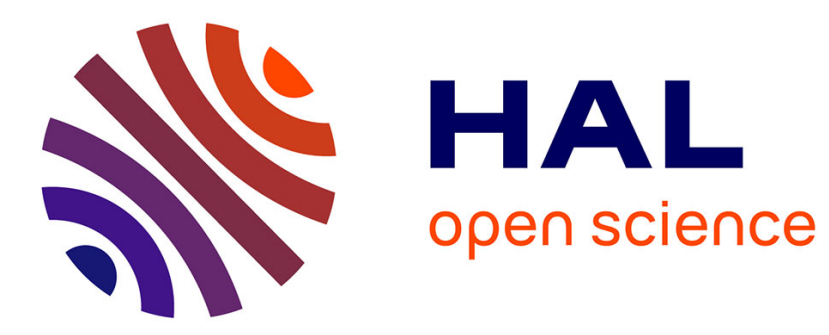

\title{
Prediction of multi-cracking in sub-micron films using the coupled criterion
}

Dominique Leguillon, Eric Martin

\section{To cite this version:}

Dominique Leguillon, Eric Martin. Prediction of multi-cracking in sub-micron films using the coupled criterion. International Journal of Fracture, 2017, pp.1-16. 10.1007/s10704-017-0255-6 . hal01675129

\section{HAL Id: hal-01675129 \\ https://hal.sorbonne-universite.fr/hal-01675129}

Submitted on 4 Jan 2018

HAL is a multi-disciplinary open access archive for the deposit and dissemination of scientific research documents, whether they are published or not. The documents may come from teaching and research institutions in France or abroad, or from public or private research centers.
L'archive ouverte pluridisciplinaire HAL, est destinée au dépôt et à la diffusion de documents scientifiques de niveau recherche, publiés ou non, émanant des établissements d'enseignement et de recherche français ou étrangers, des laboratoires publics ou privés. 


\title{
Prediction of multi-cracking in sub-micron films using the coupled criterion
}

\author{
Dominique Leguillon $^{1 *}$, Eric Martin ${ }^{2}$ \\ ${ }^{1}$ Institut Jean le Rond d'Alembert, CNRS UMR 7190, Sorbonne Universités, UPMC Université \\ Paris 06, F-75005 Paris, France. \\ ${ }^{2}$ Laboratoire des Composites Thermo-Structuraux, CNRS UMR 5801, Université de Bordeaux, \\ F-33600, Pessac, France. \\ * Corresponding author: dominique.leguillon@upmc.fr
}

\begin{abstract}
Sub-micron films deposited on a flexible substrate are now commonly used in electronic industry. The main damaging mode of these systems is a multi-cracking of the film under the action of thermal and mechanical stresses. This multi-cracking phenomenon is described using the coupled criterion based on the simultaneous fulfilment of an energy and a stress criteria. The coupled criterion is implemented in a representative volume element and it allows to decide whether the stress or the energy condition governs the cracking mechanism. It is found that the energy conditions predominates for very thin films whereas the stress condition can take place for thicker films. The initial density of cracks is determined and is in good agreement with the experimental measures. Further subdivisions, when increasing the load, are also predicted. Moreover, under some conditions, a master curve can rule the density of cracks function of the applied strain, showing a good agreement between predictions and experiments for a wide range of film thicknesses.
\end{abstract}

\section{Introduction}

Some modern electronic devices require brittle coatings deposited on flexible polymer substrates to act as anti-corrosion or gas-barrier layers (Crawford, 2005). It is obviously necessary to study the mechanical integrity of such systems (Evans and Hutchinson, 1995; Chen et al., 2002; Freund and Suresh, 2004) whose mode of degradation is a multi-cracking in the film possibly followed by a decohesion from the substrate (Bordet el al., 1998).The first obstacle that stands in the study of these systems, both experimentally (Ramsey et al., 1991) and theoretically, is linked to the extreme thinness (from some tens to some hundreds of nanometers) and brittleness of the films. The first question that arises is: do the usual fracture criteria still work in such situation? In general, an energy criterion, based on the concept of Finite Fracture Mechanics (FFM) (Hashin, 1996) is selected (Jansson et al., 2006a, Jansson et al., 2006b), but a comparison between an energy criterion and a stress criterion can also be found (Hsueh and Yanaka, 2003, Andersons et al., 2008). The first aim of this paper is to show that the coupled criterion (CC) (Leguillon, 2002; Weissgraeber et al., 2016), which enters within the field of FFM and based on the simultaneous fulfilment of the two criteria (i.e. stress and energy), also works, even for very thin films, and makes it possible to decide which one of the two conditions takes over.

The second obstacle lies in the cracking mechanism itself, multiple cracking occurs under the action of residual and mechanical stresses (bending or tensile loading). The observed parallel cracks always cut through the entire film thickness and go through the entire width of the specimen (Hsueh and Yanaka, 2003), thus a 2D model is an appropriate simplification. But despite this simplification, so high densities of cracks (hundreds of cracks per millimeter in (Yanaka et al., 1999)) are experimentally observed that it is almost impossible to carry out numerical simulations in such geometries. Facing this difficulty, many authors have opted for 
calculations in a representative volume element (RVE) (e.g. Yanaka et al., 1999 ; Hsueh and Yanaka, 2003 ; Timm et al., 2003; Jansson et al., 2006a ; Zhang et al., 2008 ; Fu et al. 2013). The same approach will be retained here. The RVE is shown in Figure 1 and the complete structure is obtained by periodically repeating this RVE. The half-length $l$ (i.e. the half-period) of the RVE is a parameter of the model intended to represent the cracks spacing, as will be seen further on. In the absence of cracks, there is no pattern to define the periodicity and any RVE half-length $l$ can be used to reconstruct the whole specimen.

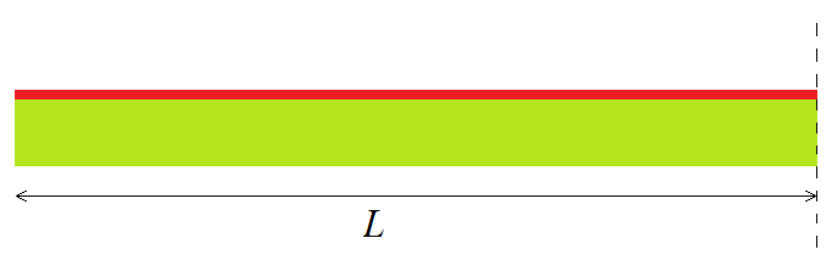

(a)

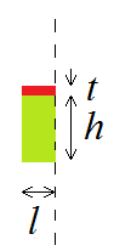

(b)

Figure 1. The coating system (a) and a corresponding RVE (b) (not at the right scale). The film is in red and the substrate in green. In both cases only one half of the frame is shown due to the symmetry axis (on the right).

The paper is outlined as follows. Section 2 is dedicated to the calculation of the thermal residual stresses in the RVE taking into account the bending effect caused by the asymmetry of the specimens. A comparison with experiments by Andersons et al. (2008) and Hsueh and Yanaka (2003) follows. In the next section, the CC is implemented to predict the nucleation of a group of cracks. Results are again compared to experiments by Andersons et al. and by Hsueh and Yanaka. Density of cracks determination is discussed in Section 4, starting with the definition of a reduced RVE. Then two cases are examined depending if a delamination takes place or not at the tip of the through-thickness cracks. Results are compared with Hsueh and Yanaka measurements.

\section{The residual stresses}

\subsection{A preliminary remark}

The first obstacle met when calculating in a RVE is the determination of the thermal residual stresses. The coating system being in general non symmetric, this results in a shrinkage and a bending of the specimen during cooling (Evans and Hutchinson, 1995). Far from the end of the specimen, the cross sections are assumed to remain straight, which makes it possible to reconstruct the whole structure by juxtaposition. Thus, the only possible motions are a translation and a rotation as depicted in Figure 2 (Leguillon et al., 2016; Leguillon et al., 2017).

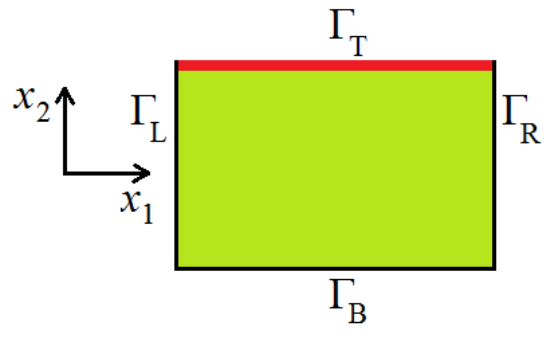

(a)

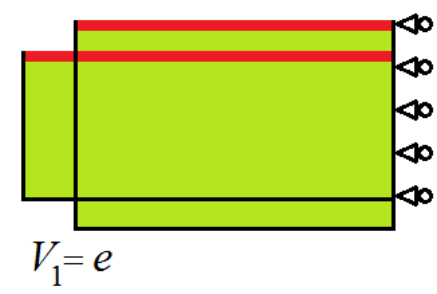

(b)

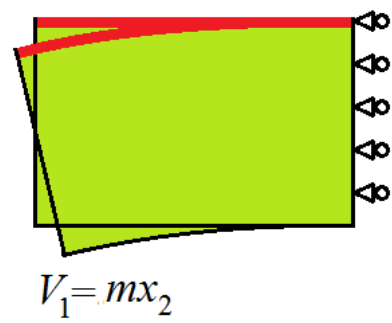

(c) 
Figure 2. The RVE (a), elongation (b) and bending (c) prescribed displacements on the left boundary. The right boundary is a symmetry axis, the top and bottom boundaries are traction free.

Within this framework, two purely elastic problems are considered: a tensile (index t) and a bending one (index b).

- The boundary conditions for the tensile problem (Figure $2 \mathrm{~b}$ ) are:

$$
V_{1}^{\mathrm{t}}=0, \sigma_{12}^{\mathrm{t}}=0 \text { on } \Gamma_{\mathrm{R}} ; \underline{\underline{\sigma^{\mathrm{t}}}} \cdot \underline{n}=0 \text { on } \Gamma_{\mathrm{B}} \text { and } \Gamma_{\mathrm{T}} ; V_{1}^{\mathrm{t}}=e \text { and } \sigma_{12}^{\mathrm{t}}=0 \text { on } \Gamma_{\mathrm{L}}
$$

where $V_{1}^{\mathrm{t}}$ is the horizontal component of the displacement field, ${\underline{\underline{\sigma^{\mathrm{t}}}}}^{\mathrm{i}}$ is the stress field and $\sigma_{12}^{\mathrm{t}}$ its shear component, $\underline{n}$ is the outward normal and $e$ a constant. Prior to the onset of a crack in the film, the solution can easily be analytically calculated (see Appendix 1).

- The boundary conditions for the bending problem (Figure 2c) are

$$
V_{1}^{\mathrm{b}}=0, \sigma_{12}^{\mathrm{b}}=0 \text { on } \Gamma_{\mathrm{R}} ; \underline{\underline{\sigma}}^{\mathrm{b}} \cdot \underline{n}=0 \text { on } \Gamma_{\mathrm{B}} \text { and } \Gamma_{\mathrm{T}} ; V_{1}^{\mathrm{b}}=m x_{2} \text { and } \sigma_{12}^{\mathrm{b}}=0 \text { on } \Gamma_{\mathrm{L}}
$$

where $m$ is another constant. Again, prior to the onset of a crack in the film, the solution is also analytically known (see Appendix 1).

Although these solutions are analytically known, we go on because the following reasoning remains valid in more complex cases computed by finite elements (FE) and especially when cracks are present in the RVE (see further on in Figures 8, 18 and 22).

Let the operator $A$ be defined for two displacements fields $\underline{U}$ and $\underline{V}$ by

$$
A(\underline{U}, \underline{V})=\int_{\Omega} \underline{\underline{\sigma}}(\underline{U}): \nabla \underline{V} \mathrm{~d} x
$$

where $\nabla$ is the gradient operator, $\underline{V}$ is one of the two solutions $\underline{V}^{\mathrm{t}}$ or $\underline{V}^{\mathrm{b}}$ and $\underline{\underline{\sigma}}(\underline{U})$ is the stress field associated with a displacement field $\underline{U}$ through Hooke's law

$$
\begin{aligned}
& \underline{\underline{\sigma}}(\underline{U})=\underline{\underline{\underline{C}}}: \nabla \underline{U} \text { for an elastic problem } \\
& \underline{\underline{\sigma}}(\underline{U})=\underline{\underline{\underline{C}}}:\left(\nabla \underline{U}-\underline{\underline{\varepsilon}}^{\text {in }}\right) \text { for a thermo-elastic problem with } \underline{\underline{\varepsilon}}^{\text {in }}=\alpha \Delta \theta \underline{\underline{I}}
\end{aligned}
$$

$\underline{\underline{\underline{\underline{C}}}}$ is the symmetric fourth order elasticity tensor, $\alpha$ the coefficient of thermal expansion (these two parameters take different values in the film and in the substrate), $\Delta \theta$ the temperature change and $\underline{\underline{I}}$ the second order identity tensor (isotropy is assumed). Thus (3) and (4) give

$$
\begin{aligned}
& A(\underline{U}, \underline{V})=\int_{\Omega} \underline{\underline{\underline{C}}}: \nabla \underline{U}: \nabla \underline{V} \mathrm{~d} x \text { or } \\
& A(\underline{U}, \underline{V})=\int_{\Omega} \underline{\underline{\underline{C}}}:\left(\nabla \underline{U}-\underline{\underline{\varepsilon}}^{\text {in }}\right): \nabla \underline{V} \mathrm{~d} x
\end{aligned}
$$


The displacement field $\underline{U}$ is solution to an elastic or thermoelastic problem with traction free top $\Gamma_{\mathrm{T}}$ and bottom $\Gamma_{\mathrm{B}}$ faces (Figure 2), with a symmetry axis along the right face $\Gamma_{\mathrm{R}}$ and in addition it is required to fulfill $\sigma_{12}(\underline{U})=0$ on $\Gamma_{\mathrm{L}}$ (in particular, it can be a solution either to the tension problem or to the bending problem or to any combination of the two). Using Green's theorem it comes (no body forces is assumed)

$$
\begin{aligned}
A(\underline{U}, \underline{V}) & =-\int_{\Omega} \nabla \cdot \underline{\underline{\sigma}}(\underline{U}) \cdot \underline{V} \mathrm{~d} x+\int_{\partial \Omega} \underline{\underline{\sigma}}(\underline{U}) \cdot \underline{n} \cdot \underline{V} \mathrm{~d} s \\
& =\int_{\Gamma_{\mathrm{L}}} \underline{\underline{\sigma}}(\underline{U}) \cdot \underline{n} \cdot \underline{V} \mathrm{~d} s=\int_{\Gamma_{\mathrm{L}}} \sigma_{1 i}(\underline{U}) V_{i} \mathrm{~d} x_{2}=\int_{\Gamma_{\mathrm{L}}} \sigma_{11}(\underline{U}) V_{1} \mathrm{~d} x_{2}
\end{aligned}
$$

Then, taking $\underline{V}=\underline{V}^{\mathrm{t}}$ leads to

$$
A\left(\underline{U}, \underline{V}^{\mathrm{t}}\right)=e \int_{\Gamma_{\mathrm{L}}} \sigma_{11}(\underline{U}) \mathrm{d} x_{2}=e R(\underline{U})
$$

$R(\underline{U})$ is the resulting force associated with $\underline{U}$.

Now taking $\underline{V}=\underline{V}^{\mathrm{b}}$ gives

$$
A\left(\underline{U}, \underline{V}^{\mathrm{b}}\right)=m \int_{\Gamma_{\mathrm{L}}} \sigma_{11}(\underline{U}) x_{2} \mathrm{~d} x_{2}=m M(\underline{U})
$$

$M(\underline{U})$ is the moment associated with $\underline{U}$. Note that, according to (5) $1, M\left(\underline{V}^{\mathrm{t}}\right)=R\left(\underline{V}^{\mathrm{b}}\right)$.

In the framework of a FE discretization, let $\mathbb{K}$ be the stiffness matrix associated with the elastic system and $\mathbf{X}$ and $\mathbf{Y}$ the vectors of nodal displacements of $\underline{U}$ and $\underline{V}$. Then (5) (elastic case) can be approximated by

$$
A(\underline{U}, \underline{V}) \approx \mathbb{K} \mathbf{X} \mathbf{Y}
$$

In the thermoelastic case, one has to note first that $\underline{U}$ is solution to the following variational problem

$$
\int_{\Omega} \underline{\underline{\underline{C}}}: \nabla \underline{U}: \nabla \underline{\varphi} \mathrm{d} x=\int_{\Omega} \underline{\underline{\underline{C}}}: \underline{\underline{\varepsilon}}^{\text {in }}: \nabla \underline{\varphi} \mathrm{d} x \quad \forall \underline{\varphi}
$$

This must hold true for any test function $\underline{\varphi}$ belonging to the appropriate space. Once discretized, (10) gives the linear system

$$
\mathbb{K} \mathbf{X}=\mathbf{B}
$$

And then $(5)_{2}$ can be written

$$
A(\underline{U}, \underline{V}) \approx \mathbb{K} \mathbf{X} \mathbf{Y}-\mathbf{B} \mathbf{Y}
$$

These algebraic operations are presumably accessible to any FE code. 


\subsection{Computation of the thermal residual stresses in the RVE}

Let us now consider the constrained thermoelastic solution $\underline{V}^{\mathrm{c}}$ fulfilling Hooke's law $(4)_{2}$ and being prevented from expanding or shrinking horizontally, i.e.

$$
V_{1}^{\mathrm{c}}=0, \sigma_{12}^{\mathrm{c}}=0 \text { on } \Gamma_{\mathrm{R}} ; \underline{\underline{\sigma}}^{\mathrm{c}} \cdot \underline{n}=0 \text { on } \Gamma_{\mathrm{B}} \text { and } \Gamma_{\mathrm{T}} ; V_{1}^{\mathrm{c}}=0 \text { and } \sigma_{12}^{\mathrm{c}}=0 \text { on } \Gamma_{\mathrm{L}}
$$

The solution is easily calculated analytically and as the coefficient of thermal expansion $\alpha$ is taken 0 in the film (see Sections 2.3 and 2.4), it holds true whatever the presence of cracks (see Appendix 1).

We assume that a good approximation of the actual thermal residual stresses, but computed in the RVE, can be expressed as the following combination

$$
\underline{U}^{\text {th }}=\underline{V}^{\mathrm{c}}+z_{1} \underline{V}^{\mathrm{t}}+z_{2} \underline{V}^{\mathrm{b}}
$$

where the two dimensionless coefficients $z_{1}$ and $z_{2}$ are such that the global resultant force and moment vanish. This is a necessary condition for representing thermal residual stresses in the absence of any external load.

$$
R\left(\underline{U}^{\text {th }}\right)=A\left(\underline{U}^{\text {th }}, \underline{V}^{\mathrm{t}}\right)=0 \text { and } M\left(\underline{U}^{\text {th }}\right)=A\left(\underline{U}^{\text {th }}, \underline{V}^{\mathrm{b}}\right)=0
$$

Then using (7) and (8) with $e=m=1$ for simplicity leads to the $2 \times 2$ system

$$
\left\{\begin{array}{c}
z_{1} R\left(\underline{V}^{\mathrm{t}}\right)+z_{2} R\left(\underline{V}^{\mathrm{b}}\right)=-R\left(\underline{V}^{\mathrm{c}}\right) \\
z_{1} M\left(\underline{V}^{\mathrm{t}}\right)+z_{2} M\left(\underline{V}^{\mathrm{b}}\right)=-M\left(\underline{V}^{\mathrm{c}}\right)
\end{array}\right.
$$

This system is either analytically known (if there is no crack in the film, Appendix 2) or can be approximated using (9) and (12) by

$$
\left\{\begin{array}{c}
z_{1} \mathbb{K} \mathbf{X}^{\mathrm{t}} \mathbf{X}^{\mathrm{t}}+z_{2} \mathbb{K} \mathbf{X}^{\mathrm{b}} \mathbf{X}^{\mathrm{t}}=\mathbf{B} \mathbf{X}^{\mathrm{t}} \\
z_{1} \mathbb{K} \mathbf{X}^{\mathrm{t}} \mathbf{X}^{\mathrm{b}}+z_{2} \mathbb{K} \mathbf{X}^{\mathrm{b}} \mathbf{X}^{\mathrm{b}}=\mathbf{B} \mathbf{X}^{\mathrm{b}}
\end{array}\right.
$$

where $\mathbf{X}^{\mathrm{t}}$ (resp. $\mathbf{X}^{\mathrm{b}}$ and $\mathbf{X}^{\mathrm{c}}$ ) is the vector of nodal displacements associated with $\underline{V}^{\mathrm{t}}$ (resp. $\underline{V}^{\mathrm{b}}$ and $\underline{U}^{\mathrm{c}}$ ). Note that in (12)

$$
\mathbb{K} \mathbf{X}^{\mathrm{c}} \mathbf{X}^{\mathrm{t}}=0 \text { and } \mathbb{K} \mathbf{X}^{\mathrm{c}} \mathbf{X}^{\mathrm{b}}=0
$$

because of the boundary conditions (13).

This is a crucial point, the system (17) makes it possible to update the residual stresses even when cracks are present.

The bending is due to the asymmetry of the specimen. The coefficient $z_{2}$ is the tangent of the angle of rotation of the left face $\Gamma_{\mathrm{L}}$ and the radius of curvature $r$ of the specimen can be derived

$$
r=\frac{l}{z_{2}}
$$


It must be emphasized that this approach a priori excludes to carry out calculations on an RVE with a reduced height as suggested in Jansson et al. (2006a) (see Section 4.1 to soften this assertion).

\subsection{Comparison with Andersons et al. experiments}

In a first step, data are taken from (Andersons et al., 2008). They carry out experiments on a coating/substrate system with different film thicknesses $t=0.1,0.2,0.4,0.6$ and $0.8 \mu \mathrm{m}$. Geometry and notations are given in Figure 1, the half-length of the whole specimen $L=2.56$ mm was realistically selected for comparing results obtained using the RVE to a complete calculation on a specimen. The substrate is a $h=125 \mu \mathrm{m}$ thick polymide, $E_{\mathrm{s}}=5.4 \mathrm{GPa}, v_{\mathrm{s}}=$ 0.3 and the coating is made of silicon nitride, $E_{\mathrm{f}}=100 \mathrm{GPa}, v_{\mathrm{f}}=0.2$ ( $E$ denotes the Young modulus and $v$ the Poisson ratio, the index s holds for substrate and $\mathrm{f}$ for film). Poisson's ratios were chosen consistently, no information being provided in the referenced paper, within a wide range their role is not essential. The residual stresses were simulated for a cooling $\Delta \theta=-200$ ${ }^{\circ} \mathrm{C}$ by assigning a coefficient of thermal expansion $\alpha$ to the substrate and 0 to the coating as suggested in (Jansson et al., 2006a). This coefficient was adjusted to $1.5510^{-4} \mathrm{~K}^{-1}$ so that the longitudinal residual stress meets the experimental measures (Figure 3). It should be noted that what is called herein thermal residual stresses may also include some chemical shrinkage phenomena, this may explain the abnormally high coefficient of thermal expansion. As expected from the analytical solutions, results are independent of the length of the RVE (for $l=1$ to $40 \mu \mathrm{m}$ ). Neglecting the correction due to the bending leads to overestimate (actually to underestimate because it is negative) the residual stresses.

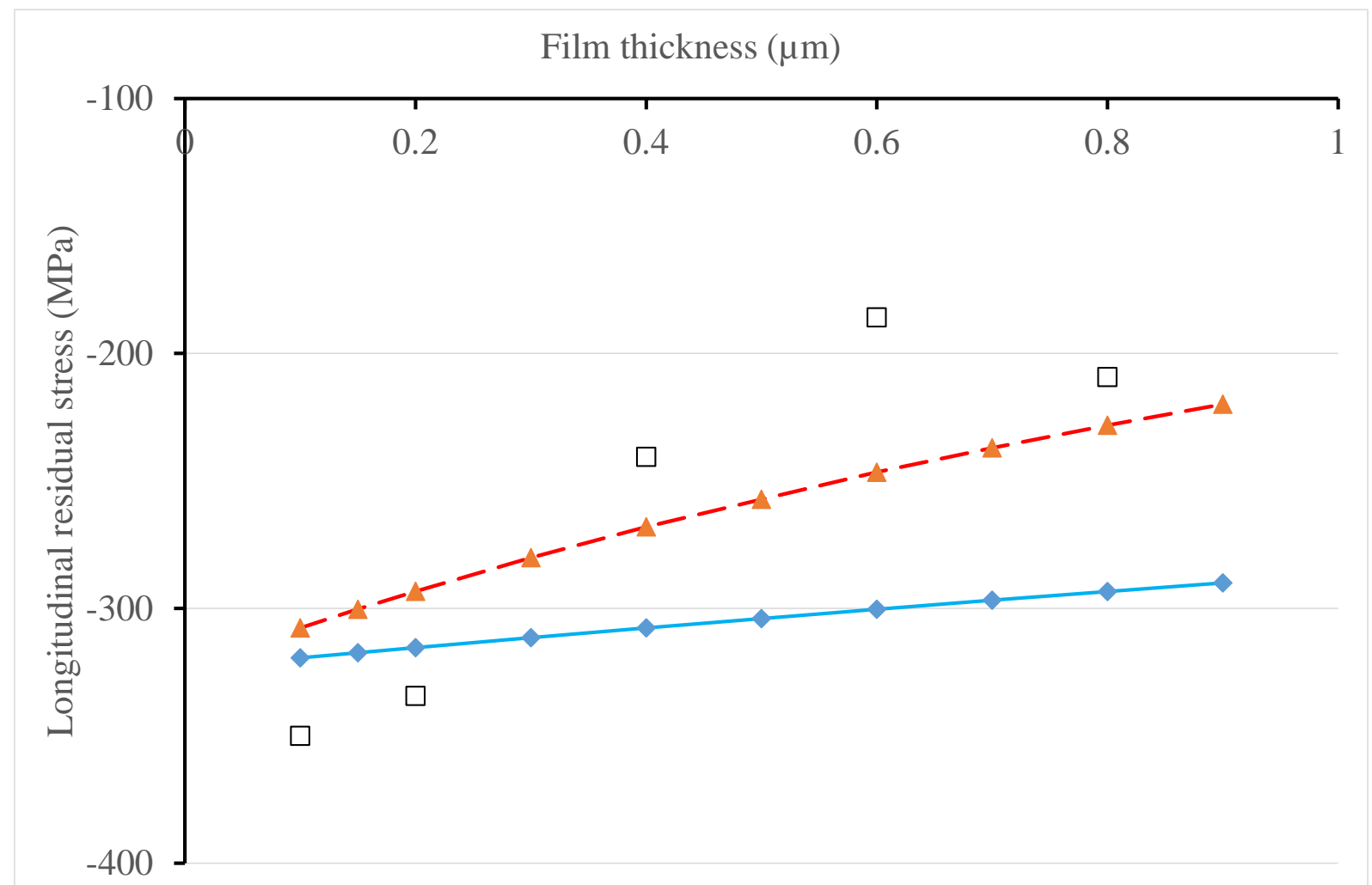

Figure 3. The longitudinal residual stress in the coating function of the coating thickness: experiments by Andersons et al. (open squares), simulation using the full correction (red dashed line and triangles), simulation neglecting the part of the correction due to the moment (blue solid line and diamonds). 
The comparison between the two components of the displacement along a cross section located at $20 \mu \mathrm{m}$ apart from the symmetry axis is shown in Figure 4. The agreement is rather good, however one has to remain very careful for this comparison because calculations in the entire specimen are not so accurate, in particular there is only one element in the thickness of the coating to make the full FE computation more tractable (a total of 186,984 DOF's) whereas there are 15 in the RVE (for a total of 32,492 DOF's).

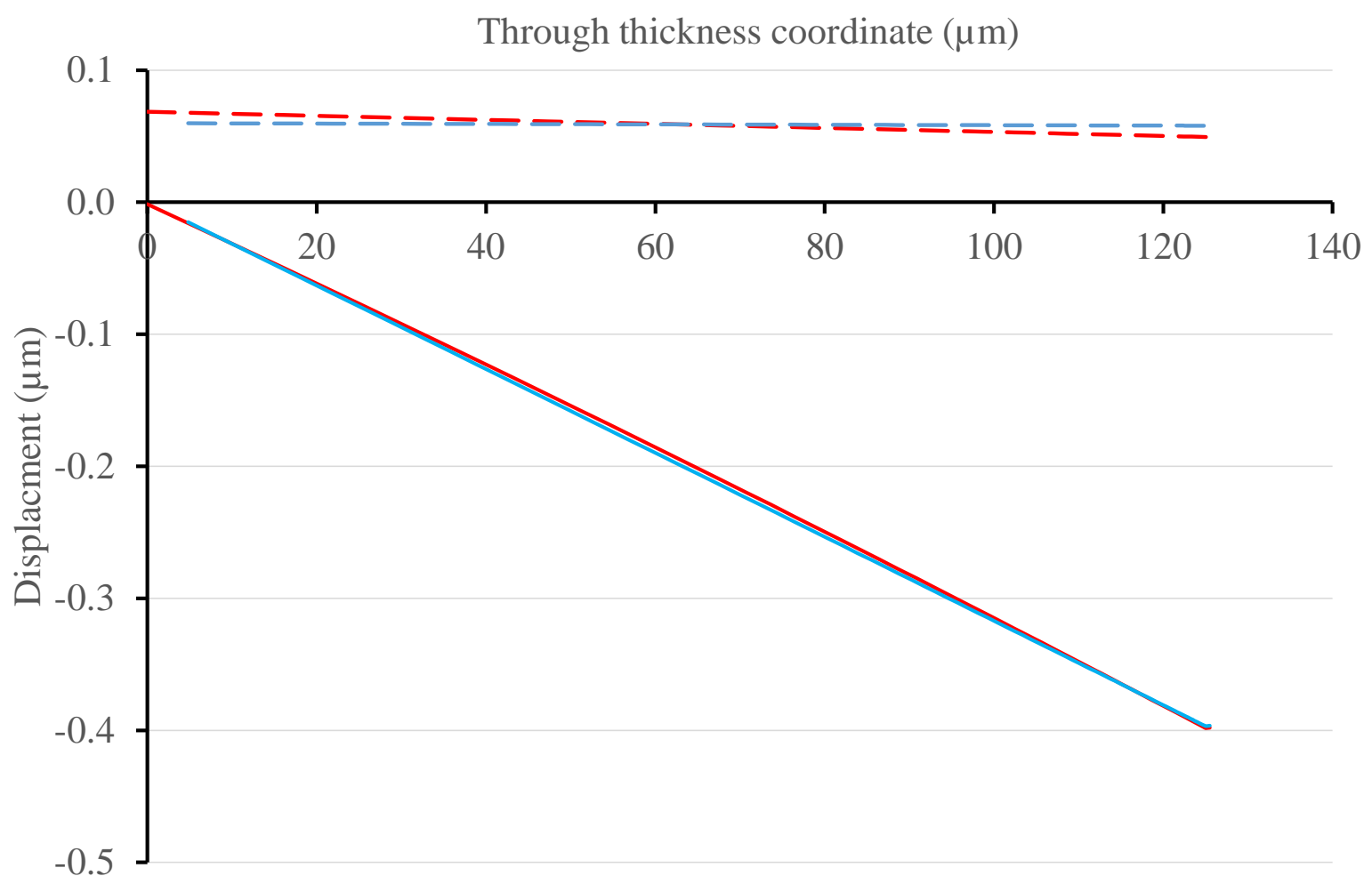

Figure 4. Comparison between displacements due to the thermal shrinkage. They are computed at $20 \mu \mathrm{m}$ of the symmetry axis in the RVE (half-length $l=20 \mu \mathrm{m}$, blue lines) and in a realistic specimen (half-length $L=2.56 \mathrm{~mm}$, red lines). The dashed lines are the horizontal components $U_{1}$ and the solid lines are the vertical components $U_{2}$.

Andersons et al. derive the residual stresses from a measurement of the curvature of the specimens after cooling due to their asymmetry. It is extremely large as a consequence of the large size of the substrate on which the film is deposited $(125 \mu \mathrm{m})$, as shown in Figure 5 using (19) (compare to Figure 6 where the substrate is thinner). Anyway, the matching between experiments and predictions is very good. 


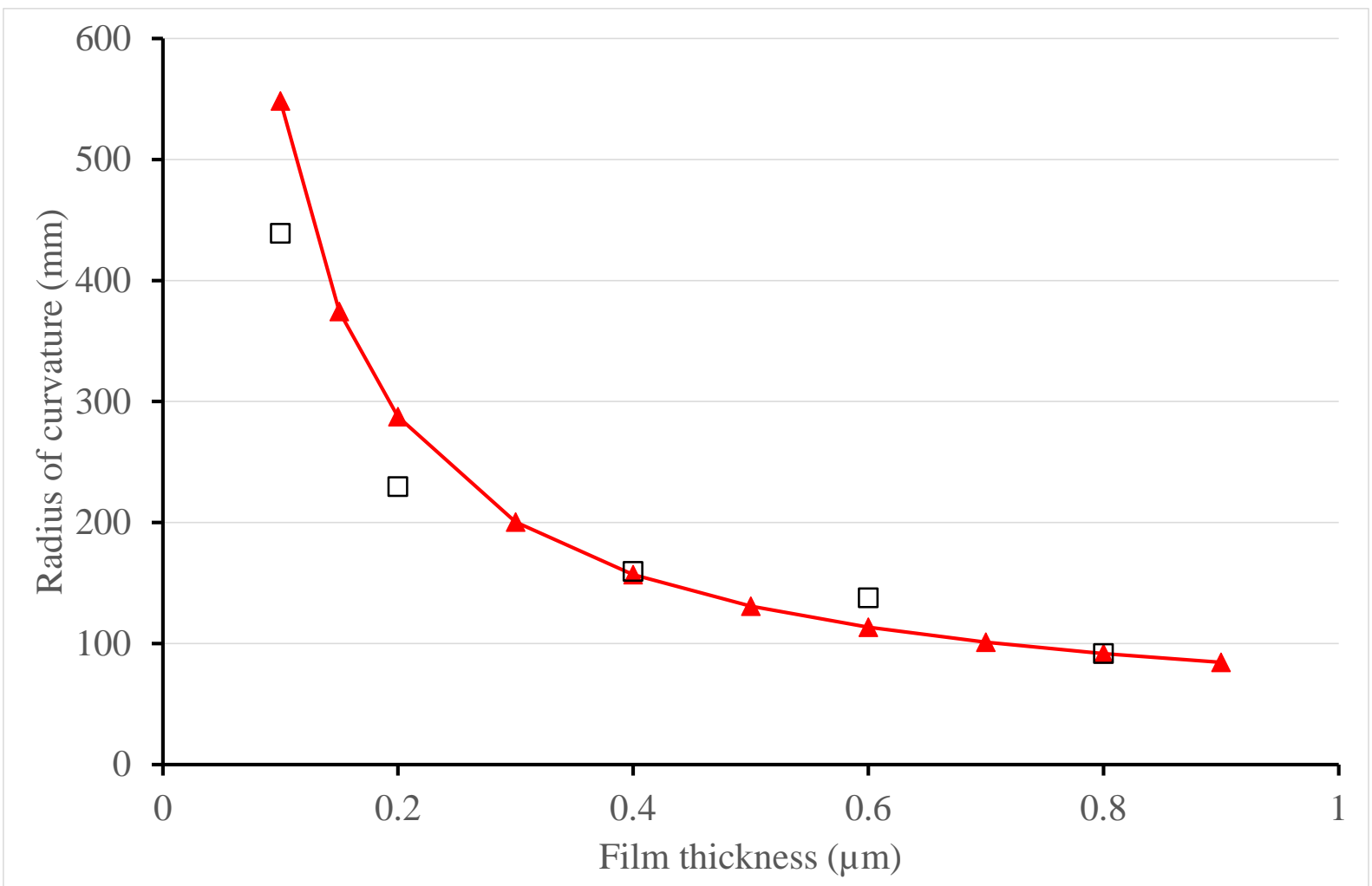

Figure 5. Radius of curvature of the specimens after cooling, function of the film thickness: prediction using (19) (red solid line and triangles), experiments by Andersons et al. (open squares).

\subsection{Comparison with Hsueh and Yanaka experiments}

Hsueh and Yanaka (2003) carried out experiments on film/substrate systems made of a $\mathrm{SiO}_{\mathrm{x}}$ coating $\left(E_{\mathrm{f}}=73 \mathrm{GPa}, v_{\mathrm{f}}=0.17\right)$ deposited by vacuum evaporation on a PET substrate $\left(E_{\mathrm{s}}=\right.$ $\left.4.84 \mathrm{GPa}, v_{\mathrm{s}}=0.35\right)$. The substrate is $h=12 \mu \mathrm{m}$ thick and various film thicknesses $t$ were experienced: 0.043, 0.067, 0.090, 0.120 and $0.320 \mu \mathrm{m}$.

Residual stresses are derived from the measurement of the curvature of the specimens after the evaporation and cooling processes (Table 1). This curvature is larger (the radius of curvature is smaller) than in the previous case (Figure 5) because the substrate is thinner. Results are expressed in the form of a mismatch strain $\Delta \varepsilon$ between the substrate and the coating. The measured radius of curvature $r=5.58 \mathrm{~mm}$ and the corresponding mismatch strain $\Delta \varepsilon=0.55 \%$ for $t=0.09 \mu \mathrm{m}$ are obviously erroneous in the referenced paper, thus the mismatch strain was smoothed to 0.43 (a radius of curvature around $7.35 \mathrm{~mm}$ ).

Table 1. The experimental measure of the radius of curvature of the specimens following evaporation and cooling, after Hsueh and Yanaka, and the resulting mismatch strain. The smoothed values are in bold.

\begin{tabular}{cccccc} 
Film thickness $t(\mu \mathrm{m})$ & 0.043 & 0.067 & 0.090 & 0.120 & 0.320 \\
\hline Radius of curvature $r(\mathrm{~mm})$ & 9.38 & 7.62 & $5.58 / 7.35$ & 7.10 & 4.69 \\
\hline Mismatch strain $\Delta \varepsilon(\%)$ & 0.59 & 0.50 & $0.55 / 0.43$ & 0.35 & 0.30
\end{tabular}

Simulations are carried out using a thermoelastic model by prescribing a virtual thermal expansion coefficient $\alpha=\Delta \varepsilon$ (Table 1) and a temperature change $\Delta \theta=-1^{\circ} \mathrm{C}$ in the substrate, whereas the film is assumed purely elastic $(\alpha=0)$. 
Using (19) allows identifying the radius of curvature $r$. A comparison showing a satisfying agreement, although tending to slightly underestimate, is proposed in Figure 6. In addition, it must be pointed out that the result is independent of the RVE half-length $l$.

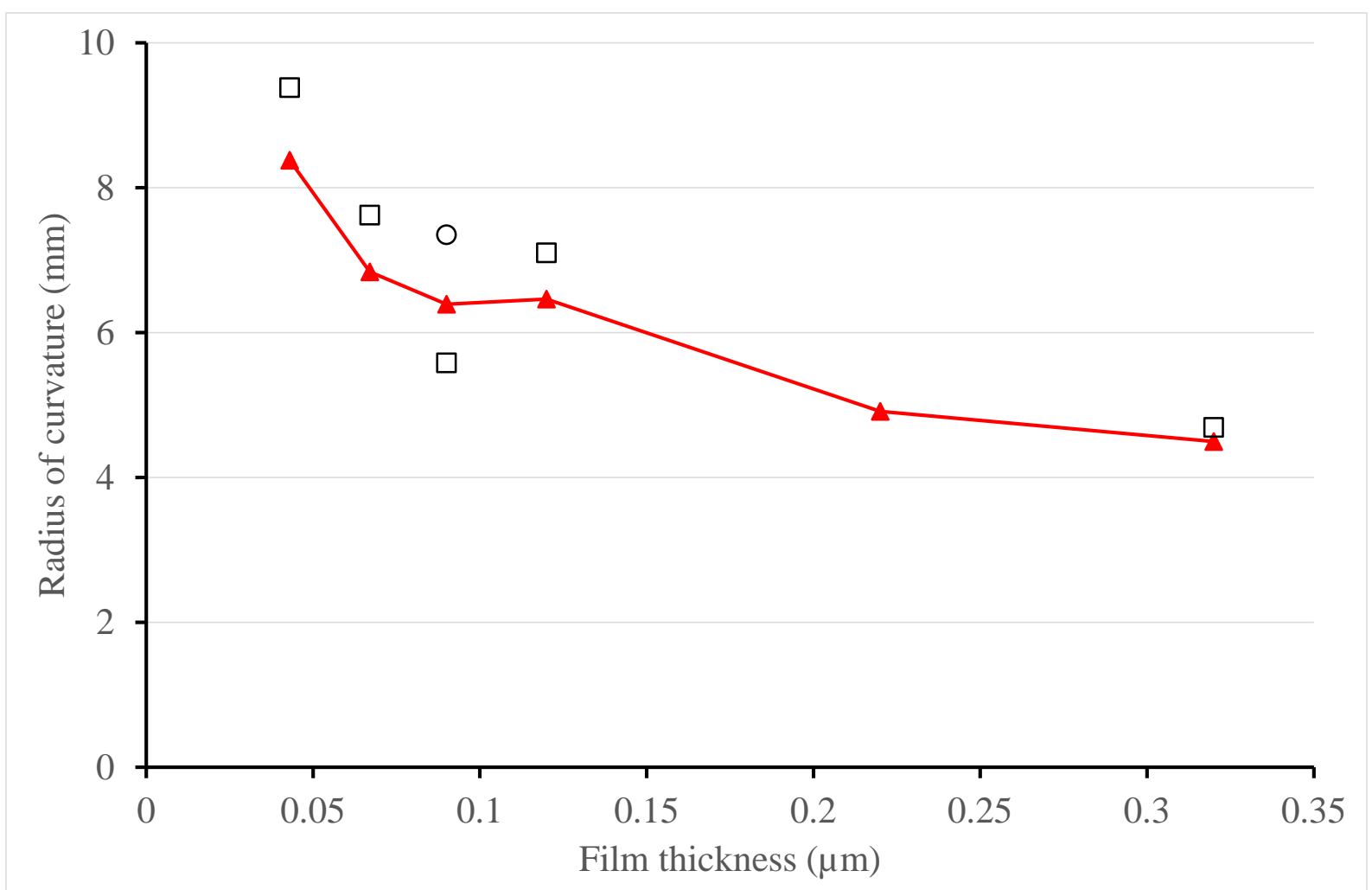

Figure 6. Comparison between the measured radius of curvature by Hsueh and Yanaka after the elaboration process (squares, the circle indicates the smoothed value) and the simulated one (red solid line and triangles).

As a consequence of the previous remark, the calculated residual stress is also independent of the RVE half-length $l$. Again neglecting the moment leads to an overestimation (Figure 7). 


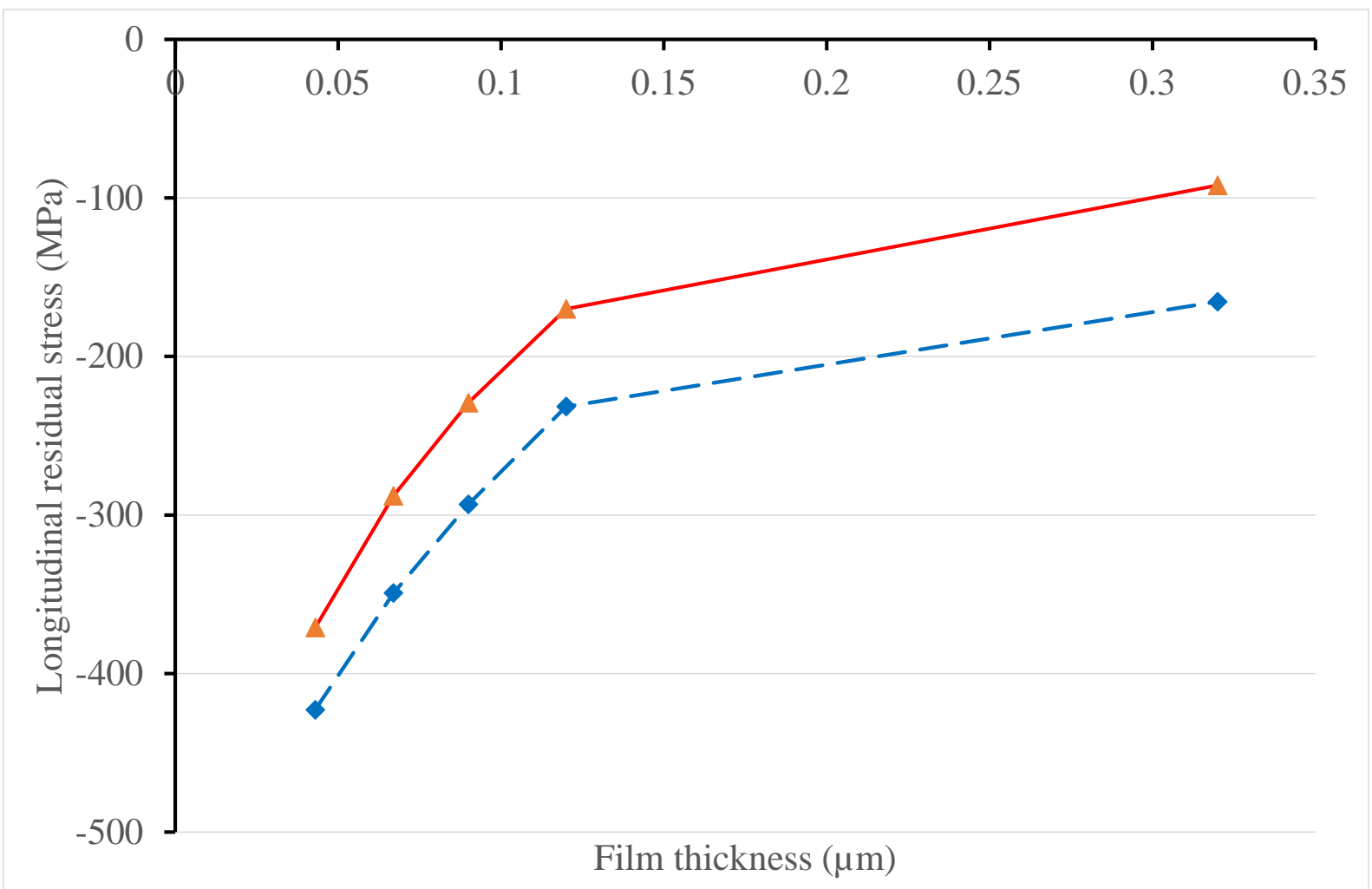

Figure 7. Residual longitudinal stress in the $\mathrm{SiO}_{\mathrm{x}}$ film after the elaboration process (red solid line and triangles) compared to the same calculation neglecting the correction due to the moment (blue dashed line and diamonds).

\section{Applied strain at the onset of cracking in the coating using the coupled criterion}

\subsection{The coupled criterion applied to the RVE}

The coupled criterion (CC) was first described in (Leguillon, 2002) and was then successfully applied to many different materials and structures (see the review paper by Weissgraeber et al. (2016)). We just briefly recall that two conditions must hold true for its fulfilment: an energy and a stress condition. It will be shown below how to use them in the case we are interested in. During the experiments, an elongation is applied to the specimen and cracking occurs in the coating at a given applied strain. Two states of the RVE are now taken into account, the uncracked state and the cracked one (Figure 8), both undergo thermal residual stresses and applied strain. The new RVE length relies on the initial density of cracks $d=1 / 2 \mathrm{l}\left(\mathrm{mm}^{-1}\right)$, this is based on the assumption that the cracks are regularly spaced as observed by Jansson et al. (2006a). 


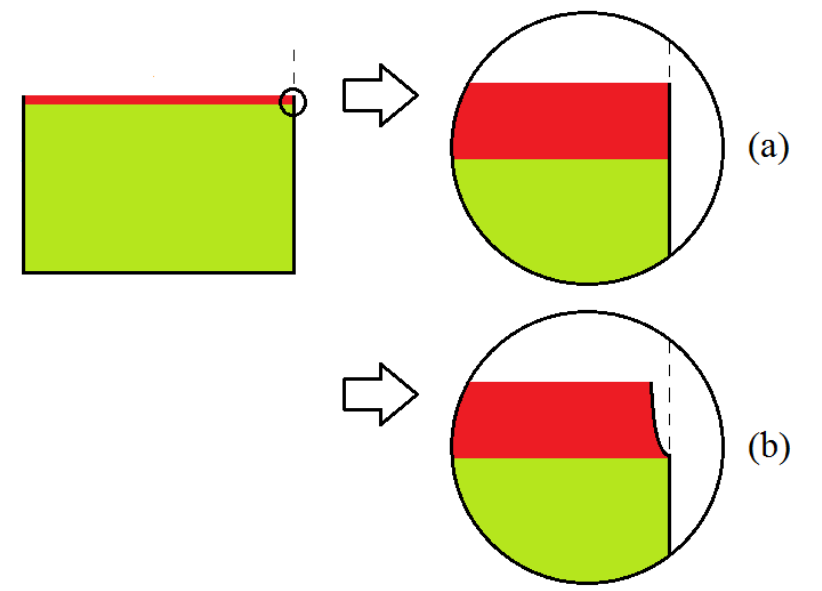

Figure 8. The two states of the RVE: (a) prior to the onset of cracks, (b) following the onset. Again, only one half of the RVE is represented with a symmetry axis on the right.

The approximations $\underline{U}$ of the solutions to these two problems are the superposition of the residual thermal stresses solution $\underline{U}^{\text {th }}$ and the elongation solution $\underline{V}^{\mathrm{t}}$ computed in the two different configurations of the RVE

$$
\underline{U}=\underline{U}^{\mathrm{th}}+f \underline{V}^{\mathrm{t}}=\underline{V}^{\mathrm{c}}+\left(f+z_{1}\right) \underline{V}^{\mathrm{t}}+z_{2} \underline{V}^{\mathrm{b}}
$$

where $f$ is the deformation of the RVE due to the applied load. The change in potential energy between these two states can be computed using the previous notations

$$
\begin{aligned}
& -\delta W^{\mathrm{P}}=-\frac{1}{2} \delta\left[\int_{\Omega} \underline{\underline{\underline{C}}}:\left(\nabla \underline{U}-\underline{\underline{\varepsilon}}^{\mathrm{in}}\right):\left(\nabla \underline{U}-\underline{\underline{\varepsilon}}^{\mathrm{in}}\right) \mathrm{d} x\right] \\
& =\delta\left[\left(f+z_{1}\right) \mathbf{B} \mathbf{X}^{\mathrm{t}}+z_{2} \mathbf{B} \mathbf{X}^{\mathrm{b}}-\frac{1}{2}\left(f+z_{1}\right)^{2} \mathbb{K} \mathbf{X}^{\mathrm{t}} \mathbf{X}^{\mathrm{t}}-\frac{1}{2} z_{2}^{2} \mathbb{K} \mathbf{X}^{\mathrm{b}} \mathbf{X}^{\mathrm{b}}-\left(f+z_{1}\right) z_{2} \mathbb{K} \mathbf{X}^{\mathrm{t}} \mathbf{X}^{\mathrm{b}}\right]
\end{aligned}
$$

The symbol $\delta$ in (21) means the change between the two states described in Figure 8. It must be pointed out that $\underline{V}^{\mathrm{c}}$ is unchanged between the two states and then $\mathrm{X}^{\mathrm{c}}$ does not appear in (21)2, while $\mathbf{X}^{\mathrm{t}}, \mathbf{X}^{\mathrm{b}}, z_{1}$ and $z_{2}$ differ in the two configurations.

The energy part of the CC can be written, in two dimensions, omitting the width of the specimen without confusion

$$
G^{\text {inc }}=-\frac{\delta W^{\mathrm{P}}}{t} \geq G_{\mathrm{c}}
$$

where $t$ is the coating thickness and the crack length and $G_{\mathrm{c}}$ the mode I toughness of the film. In (22) $G^{\text {inc }}$ is the so-called incremental energy release rate as opposed to the usual energy release rate which is differential in nature.

The stress counterpart of the CC states that the tension $\sigma_{11}$ in the film must exceed the tensile strength $\sigma_{\mathrm{c}}$ at any point of the expected crack path prior to its nucleation 


$$
\sigma_{11} \geq \sigma_{\mathrm{c}} \text { with } \sigma_{11}=\sigma_{11}(\underline{U})=\left(f+z_{1}\right) \sigma_{11}\left(\underline{V}^{\mathrm{t}}\right)+z_{2} \sigma_{11}\left(\underline{V}^{\mathrm{b}}\right)
$$

Note again that $\sigma_{11}\left(\underline{U}^{\mathrm{c}}\right)$ does not appear in (23) as a consequence of (13) and because a 0 value is assigned to the coefficient of thermal expansion $\alpha$ in the film. During a monotonic loading, i.e. an increasing value of $f$, the two inequalities (22) and (23) allows a unique determination of $f$ and the resulting applied strain $\varepsilon^{\mathrm{a}}=f / l$.

\subsection{Comparison with Andersons et al. experiments}

To compare with experiments carried out in (Andersons et al., 2008), it is worthwhile introducing what they call the intrinsic longitudinal strain $\varepsilon$ defined as

$$
\varepsilon=\varepsilon^{\mathrm{a}}+\varepsilon^{\mathrm{th}}
$$

where $\varepsilon^{\mathrm{a}}$ is the applied strain and $\varepsilon^{\text {th }}=z_{1} / l$ the strain resulting from the thermal residual stresses. Results are shown in Figure 9 and compared to experiments carried out by Andersons et al. on a silicon nitride /polymide system (material data already reported in Section 2.3) and to the Finite Fracture Mechanics (FFM) approach of Hashin (1996) as proposed by Andersons et al. As checked when solving the two inequalities deriving from the two conditions of the CC criterion, the horizontal part of the red line is entirely governed by the stress condition (23), $\sigma_{\mathrm{c}}=400 \mathrm{MPa}$ was selected to meet the experiments on this part of the curve since no information is provided in the referred paper. The growing part is governed by the energy condition (22), it is plotted for $G_{\mathrm{c}}=7 \mathrm{~J} \mathrm{~m}^{-2}$ as reported by Andersons et al. Note that Hashin's FFM criterion relies only on an energy condition. The dashed line is obtained applying the sole energy condition of the CC. 


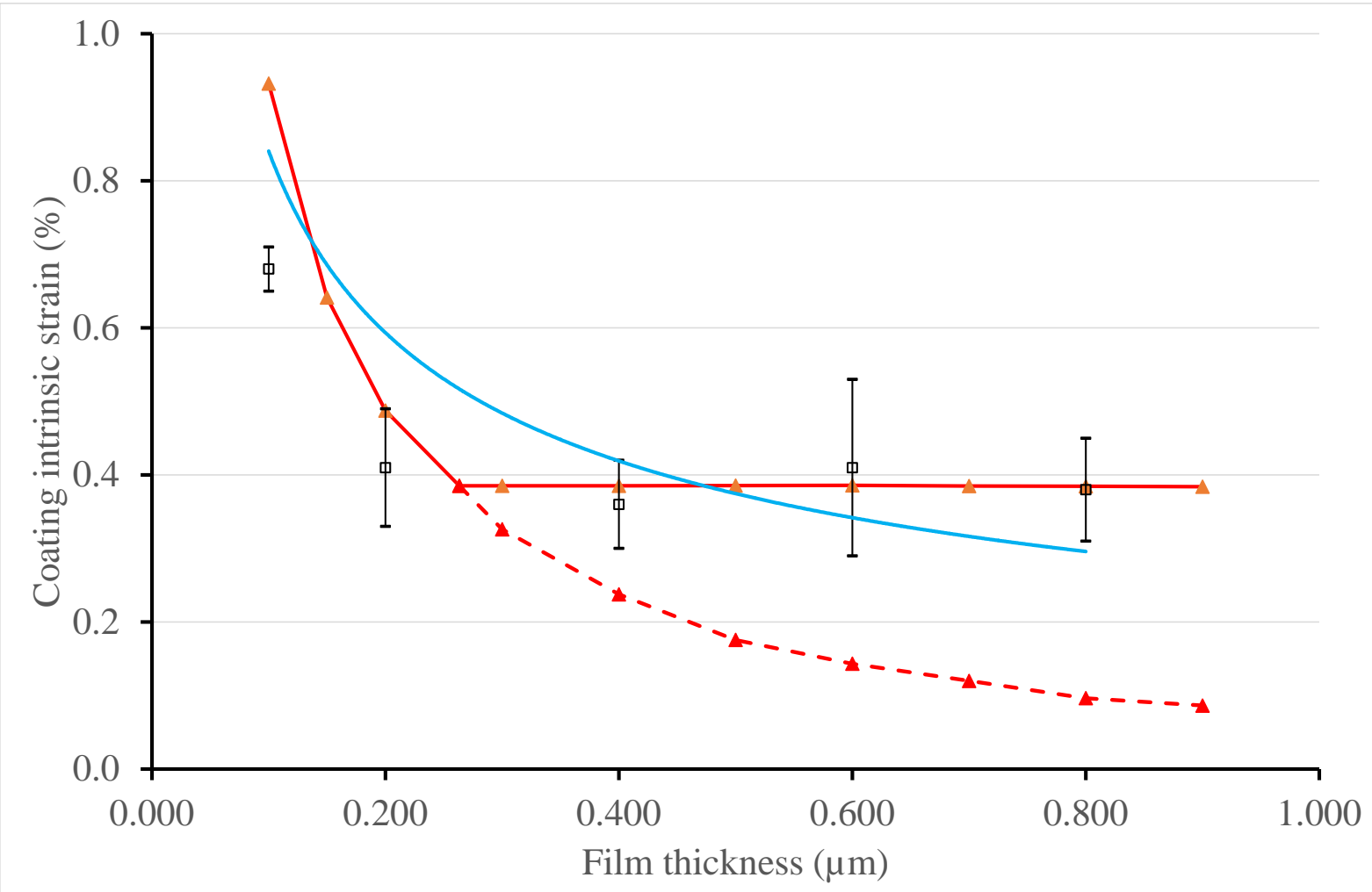

Figure 9. Intrinsic strain at the onset of cracking in the coating for $G_{\mathrm{c}}=7 \mathrm{~J} \mathrm{~m}^{-2}$. Comparison between the present prediction (red line and triangles), the experiments (squares with error bars), and prediction based on the FFM approach of Hashin (blue line) as proposed by Andersons et al.). The red dashed line is obtained applying the sole energy condition of the CC.

A better fit is obtained for $G_{\mathrm{c}}=5 \mathrm{~J} \mathrm{~m}^{-2}$ (Figure 10), however, there is only two points to adjust this value. Of course, it has no influence on the part of the curve governed by the stress condition, this may just shift the transition between the two conditions. 


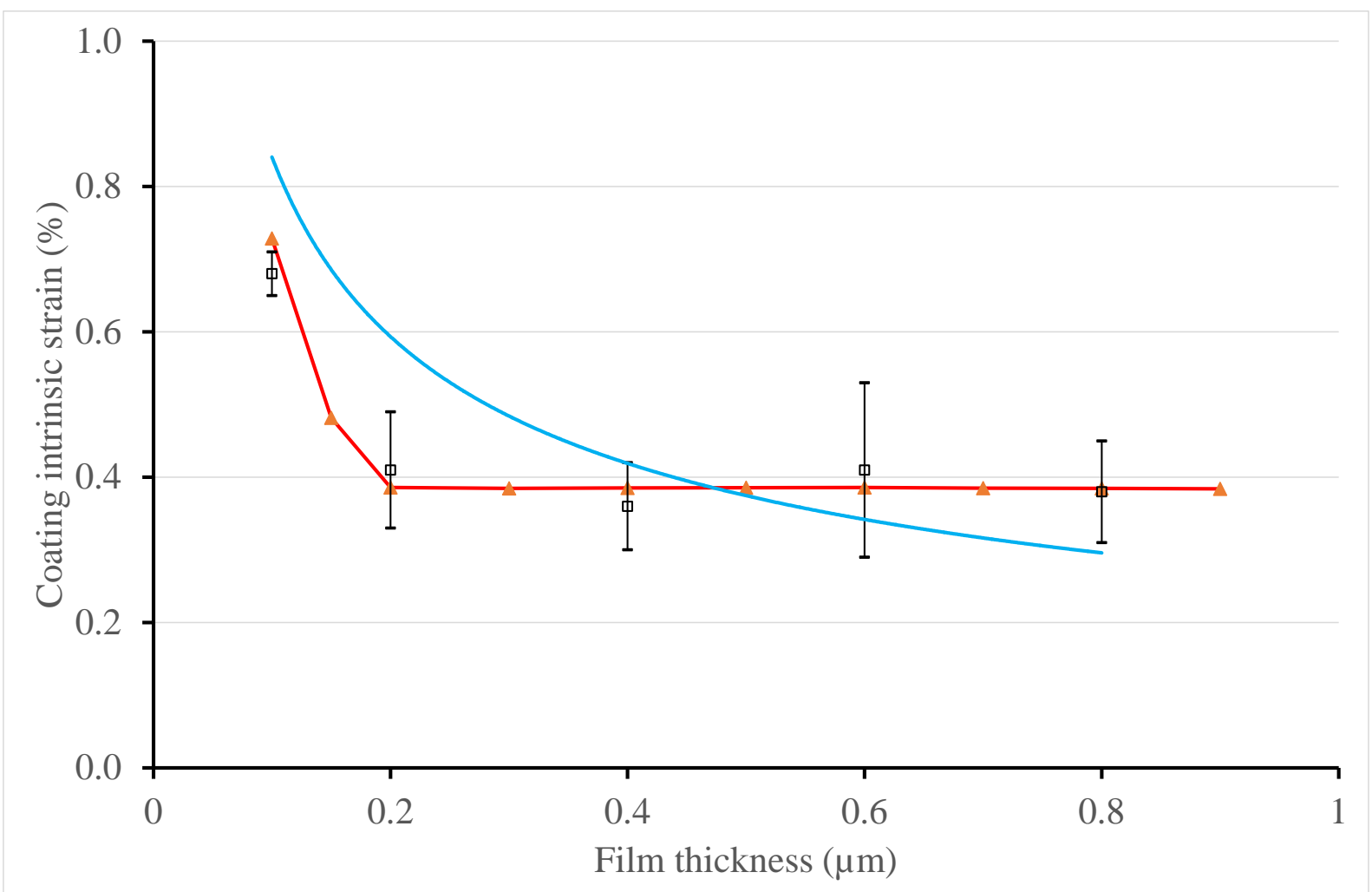

Figure 10. Intrinsic strain at failure in the film for $G_{c}=5 \mathrm{~J} \mathrm{~m}^{-2}$. Comparison between the present prediction (red line and triangles) and the experiments (squares with error bars). Refer to Figure 9 for the other symbols.

Using the intrinsic coating strain allows a comparison with the experimental results of Andersons et al., however, the applied strain at failure $\varepsilon^{\mathrm{a}}$ in (24) gives a better representation of what occurs during the experiments. The rotation tends to release the residual stresses compared to the situation where bending is inhibited. Overestimating them by neglecting the moment correction (Figure 3) leads to overestimate the load at failure when the stress condition governs and slightly shifts the transition between stress and energy driven phases, as shown in Figure 11. It must be emphasized that, as expected regarding the intrinsic strain, the result remains unchanged when the stress condition governs.

However, it can be noted that the correction brought by the moment is not very large, indeed the curvature of the specimens after cooling remains small because of the large thickness of the substrate. Contrarily, completely neglecting the residual stresses leads to a significant change (Figure 11). 


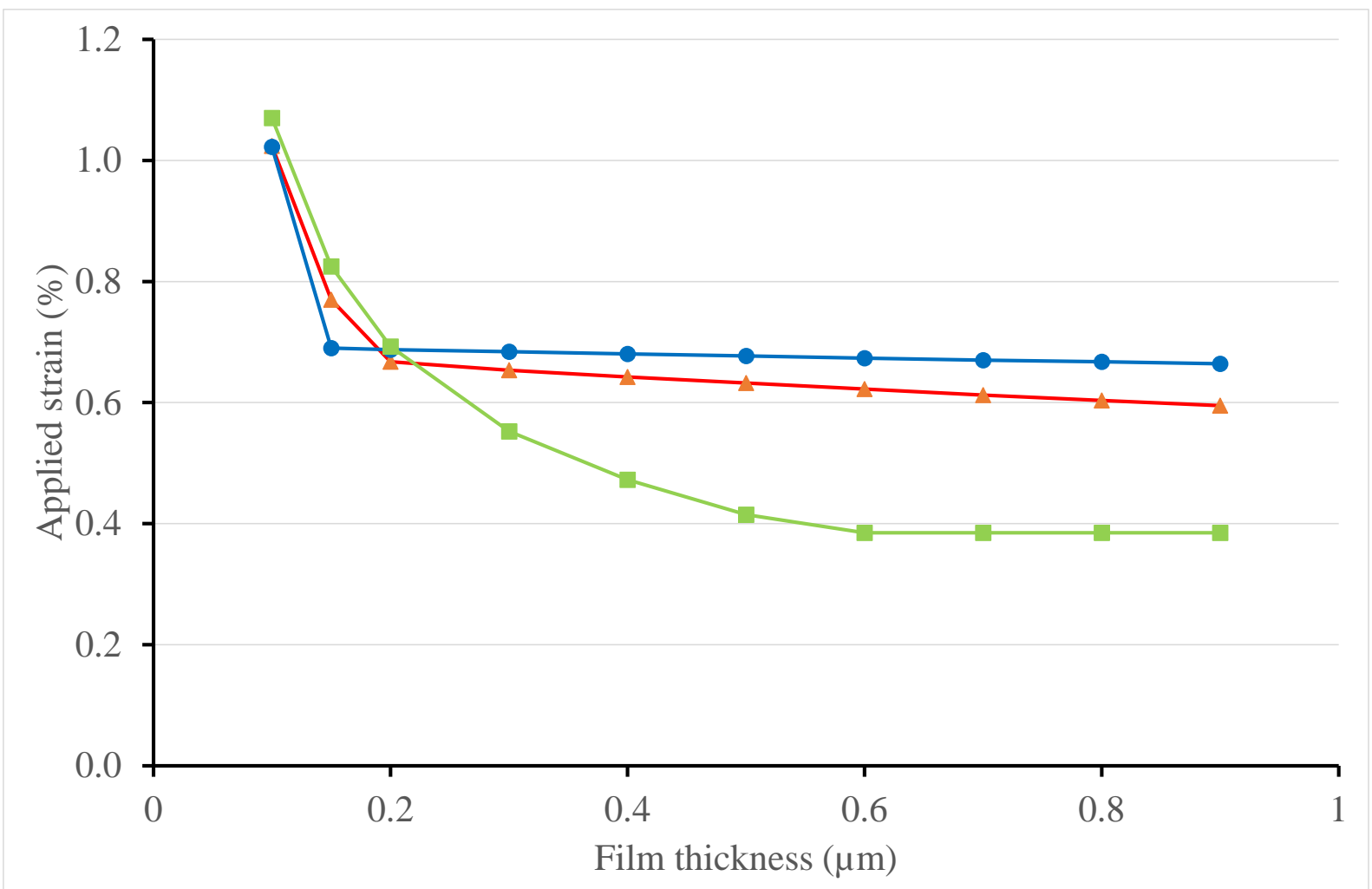

Figure 11. Applied strain at failure, comparison between the prediction based on a full calculation (red solid line and triangles), a simplified calculation neglecting the moment correction (blue solid line and circles) and a calculation omitting the residual stresses (green solid line and squares).

\subsection{Comparison with Hsueh and Yanaka experiments}

Assuming reasonably (see the remark accompanying Figure 13 below) that the tensile strength of $\mathrm{SiO}_{\mathrm{x}}$ is smaller than $400 \mathrm{MPa}$ (it is usually reported to be between 100 and $200 \mathrm{MPa}$ ), the cracking mechanism is entirely governed by the energy condition, as observed when solving the two inequalities deriving from the two conditions of the CC criterion. This is due to the thinness of the films. The fracture energy is reported to lie between 4 and $10 \mathrm{Jm}^{-2}$ in their paper, the value $G_{\mathrm{c}}=6 \mathrm{Jm}^{-2}$ was selected to best match the results. A comparison between theoretical predictions and experiments are shown in Figure 12 for RVE lengths varying from 5 to $15 \mu \mathrm{m}$. 


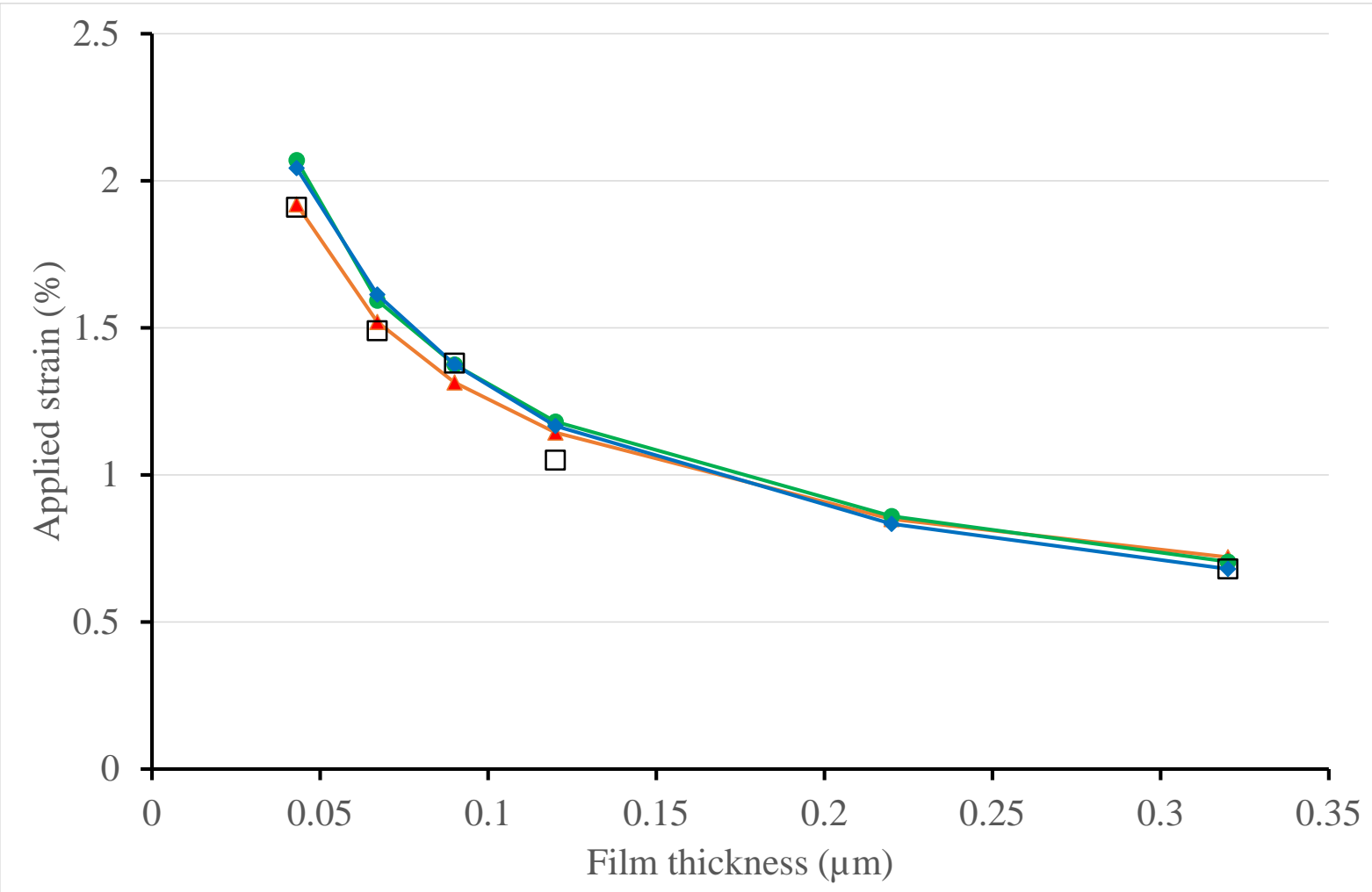

Figure 12. Theoretical predictions of the applied strain at first appearance of cracks $(l=5 \mu \mathrm{m}$, red solid line and triangles; $l=10 \mu \mathrm{m}$, green solid line and circles; $l=15 \mu \mathrm{m}$, blue solid line and diamonds) compared to the experiments of Hsueh and Yanaka (squares) with $G_{\mathrm{c}}=6 \mathrm{Jm}^{-2}$.

It is difficult to decide if the slight discrepancy, observed in Figure 12 for $l=5 \mu \mathrm{m}$ and small film thicknesses, is due to a bias of the FE mesh or not.

Hsueh and Yanaka proposed also an estimation of what they called the film strength $\sigma_{\text {str }}$, i.e. the tension which prevails in the film at the appearance of cracking. A comparison is proposed in Figure 13 with the value derived from the CC, although it is highly questionable to derive such a parameter from a mechanism governed by the energy condition. Thus, it is difficult to discuss the observed difference. However, a comment can be made that further weakens a little more the concept of film strength in the present case. Even if the tensile strength is not strictly an intrinsic parameter of the material and may depend in some way on the thickness, this dependence is probably relatively small and certainly not as important as that depicted in Figure 13: from 2.3 (CC) to 3.5 (Hsueh and Yanaka) times larger for thin films $(0.043 \mu \mathrm{m})$ than for thick ones $(0.32 \mu \mathrm{m})$. If one tries to use the statistical theory of Weibull where thinner layers are stronger than thicker ones, such ratios would be associated with very small and rather unrealistic Weibull modules ranging respectively from 2.4 to 1.6. Moreover, an observation by Andersons et al. (2008) (although on a different system) shows that the defect density is higher in thin films that could lead to an inverse conclusion to that of Weibull's theory. 


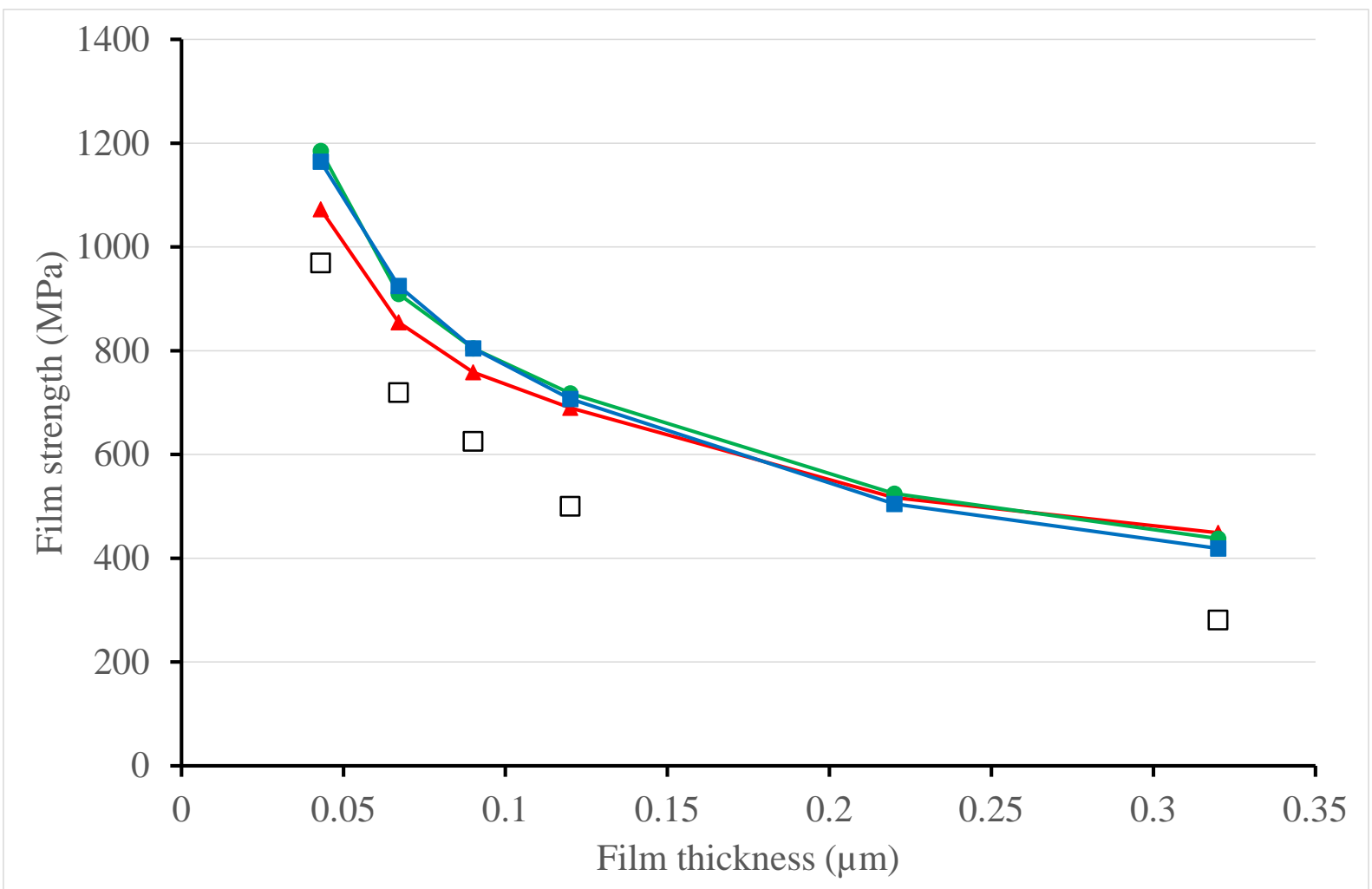

Figure 13. A comparison on the questionable concept of film strength derived either from the CC $(l=5 \mu \mathrm{m}$, red solid line and triangles; $l=10 \mu \mathrm{m}$, green solid line and circles; $l=15 \mu \mathrm{m}$, blue solid line and diamonds), or from Hsueh and Yanaka measurements (open squares).

There is no reference here to the material strength of $\mathrm{SiO}_{\mathrm{x}}$ forming the film, it is realistically supposed to be smaller than $\sigma_{\mathrm{c}}=400 \mathrm{MPa}$ and then it plays no role in the prediction of failure using the CC, because the stress condition is exceeded for the considered loadings, as can be observed in Figure 13. The material strength would have to be greater than $400 \mathrm{MPa}$ for the CC to be governed by the stress condition for $t=0.32 \mu \mathrm{m}$. It should even exceed $1100 \mathrm{MPa}$ for this to hold true for all thicknesses.

As already mentioned, the values of $z_{1}$ and $z_{2}$, solutions to (17) are updated for each density of cracks, i.e. for each RVE length $l$. This makes it possible to calculate, for example, the evolution of the radius of curvature $r$ of the specimens as a function of the density of cracks as shown in Fig. 14. This radius increases approximately linearly with the density of cracks, i.e. the curvature tends to decrease. 


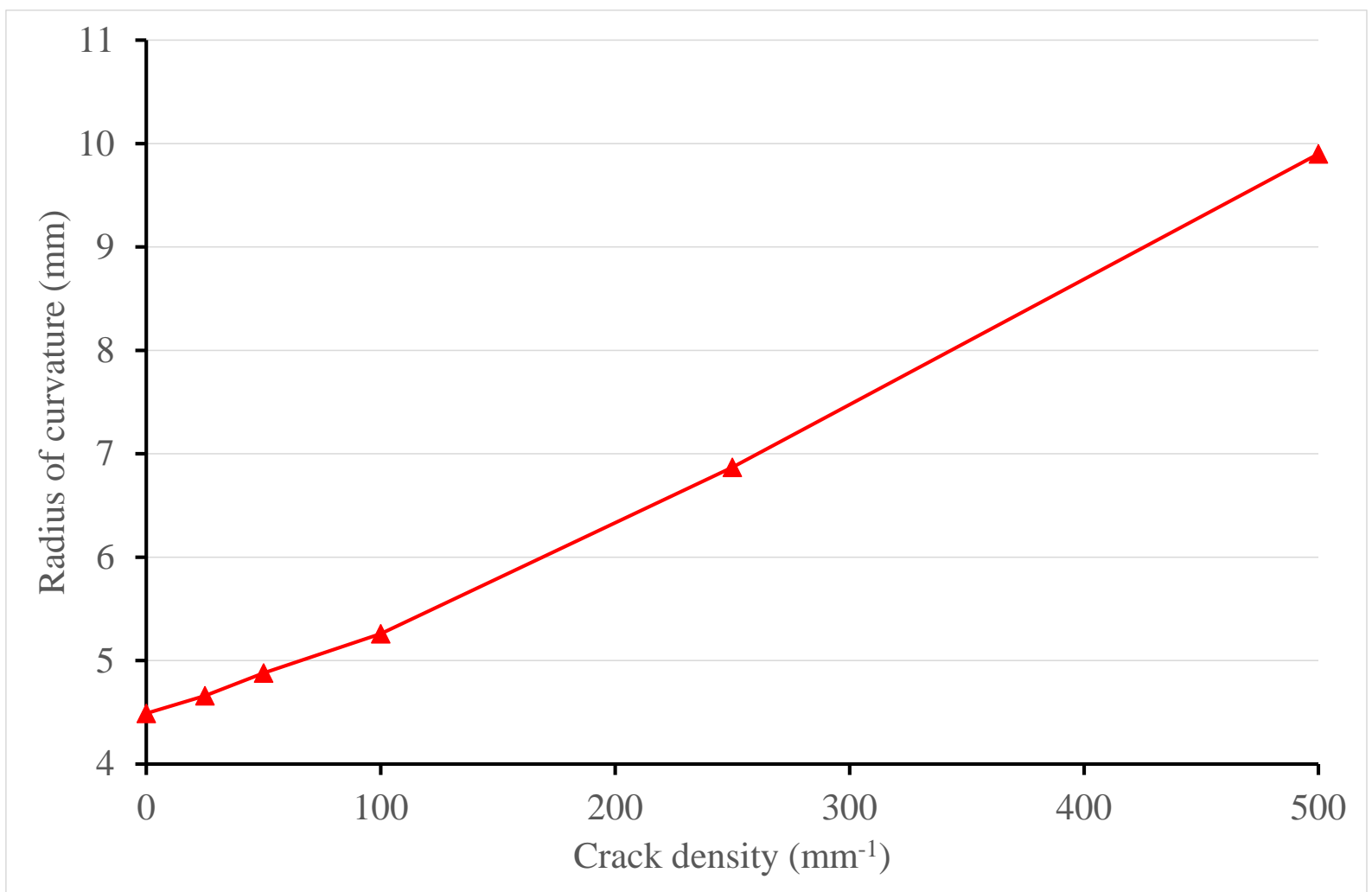

Figure 14. The radius of curvature of the specimens function of the density of cracks for $t=$ $0.32 \mu \mathrm{m}$.

\section{Determination of the density of cracks - A simplified procedure}

\subsection{Definition of a reduced $R V E$}

In Figure 1, the parameter $l$ holds for the crack spacing at the first appearance of cracking. So as to implement the iterative method described in Leguillon et al. (2017) to predict the initial density of cracks and how it evolves, it proves useful to vary this length and more precisely to shorten it. The main obstacle encountered when attempting this is the disproportion between the required RVE length and the films thickness on the one hand and the height of the substrate on the other hand. To this aim, a reduced RVE (RRVE) is used as suggested in Jansson et al. (2006a) where they replaced the total height of the RVE $(0.35 \mu \mathrm{m}$ film $+100 \mu \mathrm{m}$ substrate) by a smaller one where the substrate is only $25 \mu \mathrm{m}$ thick. In addition, they assume that the new virtual boundary at the bottom is traction free.

In order to carry out a similar reduction whatever the film thickness, templates of FE meshes are made such that $t_{0}=1, h_{0}=20 t_{0}$ and $l_{0}=\lambda \times h_{0}, \lambda / 2 \times h_{0}, \lambda / 4 \times h_{0}, \ldots$ (units are meaningless in the templates, see Figure 1 for the notations). The aspect ratio $\lambda$ is taken equal to 2 , which is sufficient for our purpose as seen later. Then these templates are scaled by $t$ to match the current film thickness. Following Jansson et al. (2006a), a traction free boundary condition is prescribed on the bottom face.

Unfortunately, such a procedure prevents to use the method proposed in Section 1.2 to estimate the residual stresses. The coefficient $z_{1}$ is approximated considering that the system (17) is uncoupled, giving

$$
z_{1}^{\prime}=\frac{\mathbf{B} \mathbf{X}^{\mathrm{t}}}{\mathbb{K} \mathbf{X}^{\mathrm{t}} \mathbf{X}^{\mathrm{t}}}
$$


where of course the matrix and the vectors involved in (25) are recalculated in the new RRVE geometry. But as the ratio between the film and the substrate thicknesses is not preserved when reducing the RVE, a correction must be brought. It is derived from the formula giving the residual longitudinal stress in the components of a bimaterial laminate (see for instance Leguillon et al., 2015)

$$
z_{1}=z_{1}^{\prime} \frac{\frac{E_{\mathrm{f}}^{*}}{E_{\mathrm{s}}^{*}} \frac{1}{h_{0}}+1}{\frac{E_{\mathrm{f}}^{*}}{E_{\mathrm{s}}^{*}} \frac{t}{h}+1} \text { where } E_{j}^{*}=\frac{E_{j}}{\left(1-v_{j}\right)} \text { with } j=\text { f or s }
$$

Note that this formula holds true only for symmetric laminates, it is used herein as an approximation which proves satisfactory.

As well, $z_{2}$ cannot be determined using (17) because the moment associated with the bending cannot be calculated in the RRVE. However, if the curvature radius is known, it can be directly used to prescribe the rotation of the left boundary (see Figure 2c). This curvature radius is often measured when there is no crack, it is even used to determine the initial state of residual stresses. However the curvature evolves with the density of cracks, but it is not reported in the experiments. It can be predicted as explained in Section 3.3 (Figure 14).

\subsection{Comparison with Hsueh and Yanaka experiments}

The comparisons and tests will be carried out only in the case of Hsueh and Yanaka experiments (2003), since no information on crack densities is provided in the case of Andersons et al. (2008).

The above simplified approach seems satisfactory when estimating the residual stresses as seen in Figure 15. Again, neglecting the bending effect leads to overestimate the residual stresses as already observed in Figure 7. 


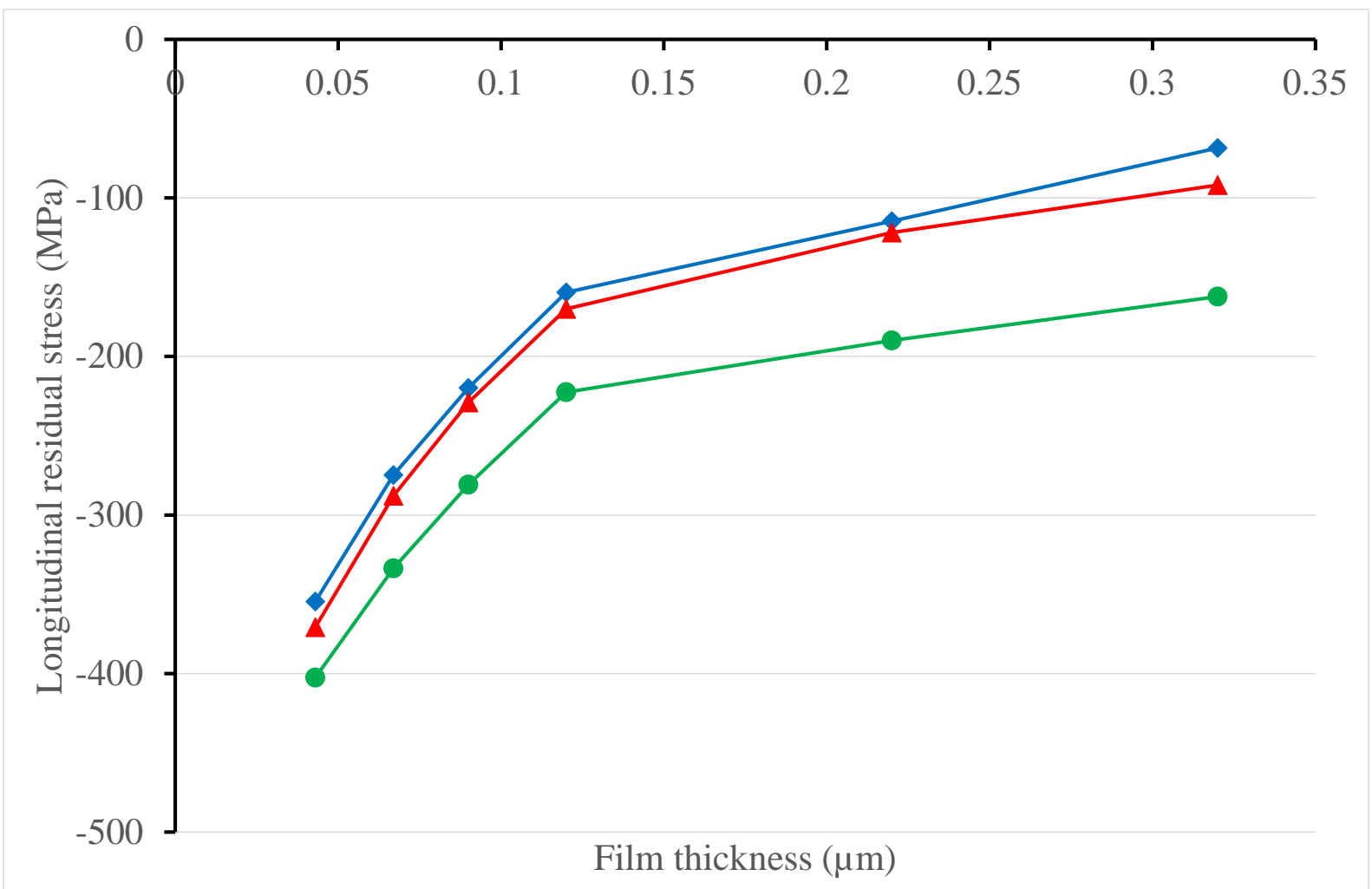

Figure 15. The longitudinal residual stress calculated using the full RVE (red solid line and triangles), using the RRVE and the bending effect (blue solid line and diamonds) and using the RRVE but ignoring the bending effect (green solid line and circles).

However, these precautions will prove unnecessary because in this case it is possible to totally neglect the residual stresses $\left(z_{1}=z_{2}=0\right)$ and carry out very simplified calculations. This is mainly because the energy criterion is governing the cracking process, as already observed. Relation (21) giving the change in potential energy, now calculated in the RRVE, reduces to

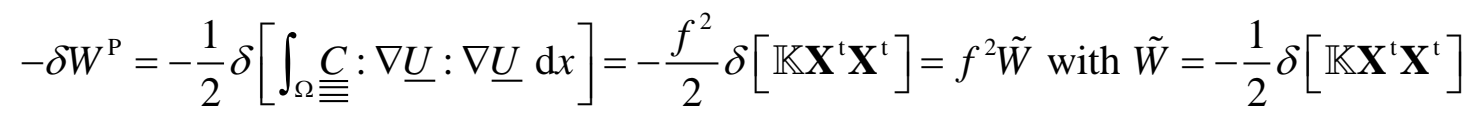

Moreover, this calculation is even more simplified because it is independent of the film thickness. It is easily shown that $\tilde{W}$ in (27) depends only on the aspect ratio $\lambda$ of the RRVE. Indeed, between two film thicknesses $t_{1}$ and $t_{2}$ the domain is scaled by the ratio $t_{2} / t_{1}$ while the strain and stress fields resulting of the unit applied deformation are scaled by $t_{1} / t_{2}$ (more precisely, the squares of these ratios intervene in (27)). As a consequence $\varepsilon^{\text {a }}$ at failure varies like $1 / \sqrt{t}$. Thus the calculation can be done once and for all for a given aspect ratio $\lambda$ and rescaled for the various film thicknesses.

Figure 16 compares the prediction of the applied strain in the case of calculations in the full RVE (Section 2.3) with $l=5 \mu \mathrm{m}$ taking into account or ignoring the residual stresses to simplified calculations in the RRVE ignoring the residual stresses. 


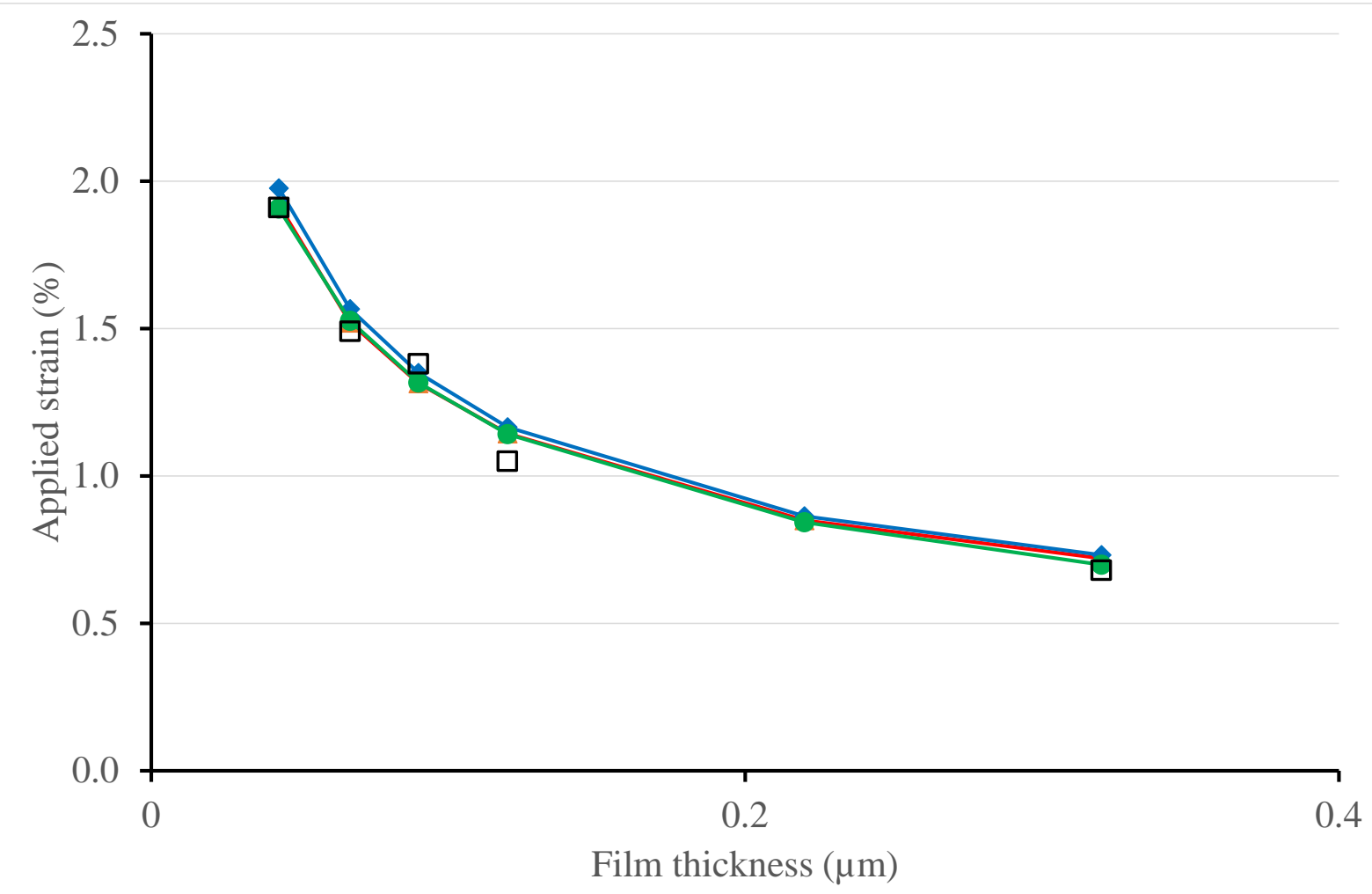

Figure 16. Theoretical predictions of the applied strain at first appearance of cracks: comparison between the calculations in the full RVE for $l=5 \mu \mathrm{m}$ taking into account the residual stresses (red solid line and triangles) and neglecting them (blue solid line and diamonds), and calculations in the RRVE again neglecting the residual stresses (green solid line and circles). The squares are the experiments by Hsueh and Yanaka.

In Figure 16, taking or not into account the residual stresses leads to neighboring curves whereas the residual stresses are far from being negligible (Figure 15). The main reason is that, as already seen, the cracking mechanism is energy driven and the state of stress plays only a minor role. Moreover, using the RRVE does not seem to deteriorate the results. These are key points allowing very simplified calculations in the forthcoming sub-sections.

\subsection{Determination of the initial density of cracks}

The prediction for the nucleation of the first group of cracks is plotted in Figure 17 for various RRVE half-length, varying $\lambda$ from $4(l=80 \times t)$ to $0.125(l=2.5 \times t)$. Obviously it is difficult to distinguish the two first curves corresponding respectively to $80 \times t$ and $40 \times t$, but for smaller values the load that causes cracking increases gradually as $l$ decreases. This means that the maximum initial density of cracks is approximately defined by $d=1 / 2 l$ with $l=40 \times t$. This gives respectively $d \approx 39,104,139,187,291 \mathrm{~mm}^{-1}$ for $t=0.32,0.12,0.09,0.067,0.043 \mu \mathrm{m}$, they are rather realistic values regarding Figures 19 and 24. 


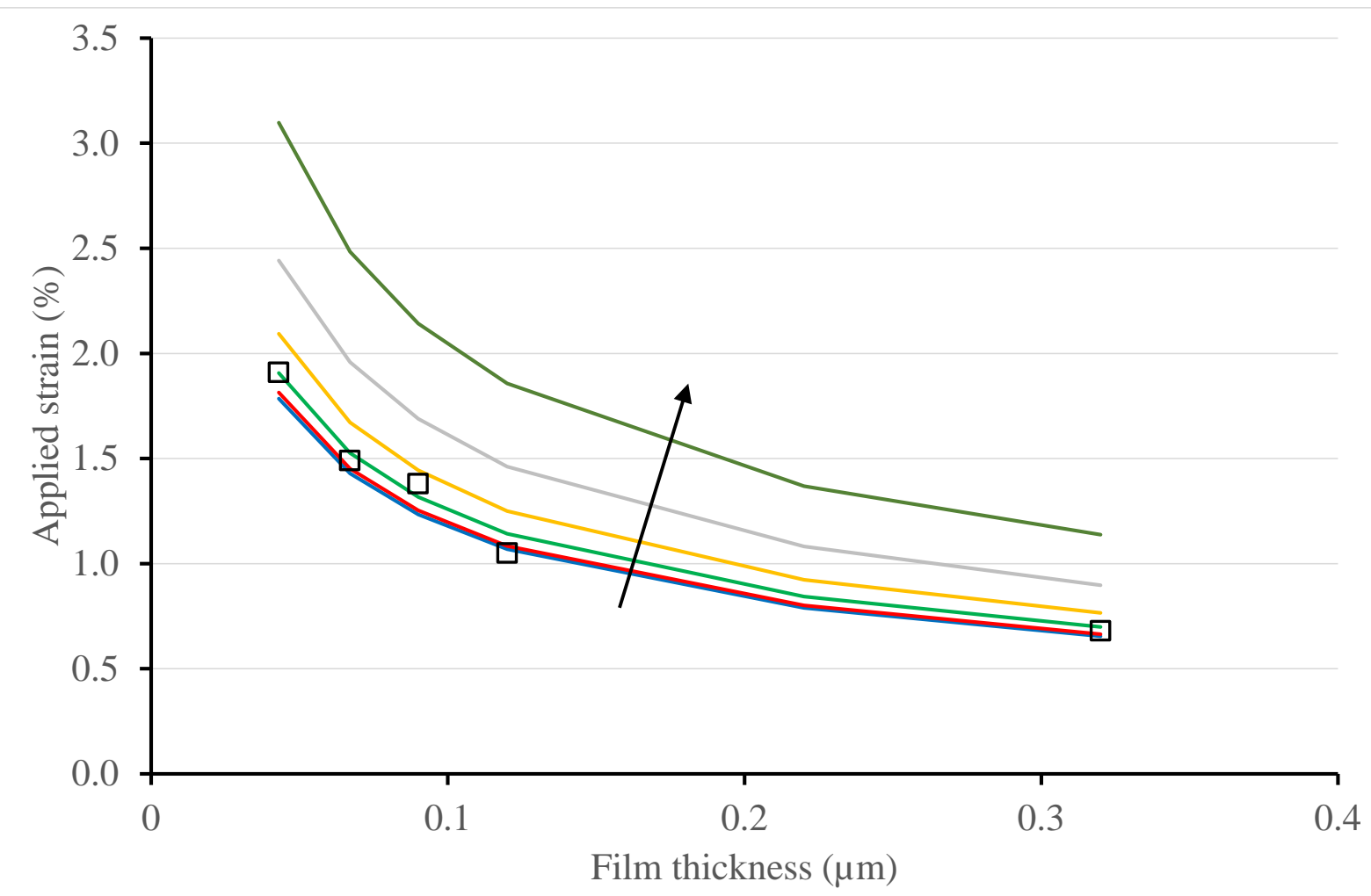

Figure 17. The predicted applied strain at failure function of the film thickness for decreasing RRVE half-length $l$ from $80 \times t$ to $2.5 \times t$, following the arrow (it is divided by 2 for each curve, starting from $80 \times t$ ).

\subsection{Forthcoming crack densities function of the applied strain, without delamination}

Once the first group of cracks is created, the RRVE is redefined as the segment located between two cracks. At the first step, it has still the same length but with one crack at each end and a new one appearing in the middle (Figure 18). At the next step its length is divided by 2 and so on.

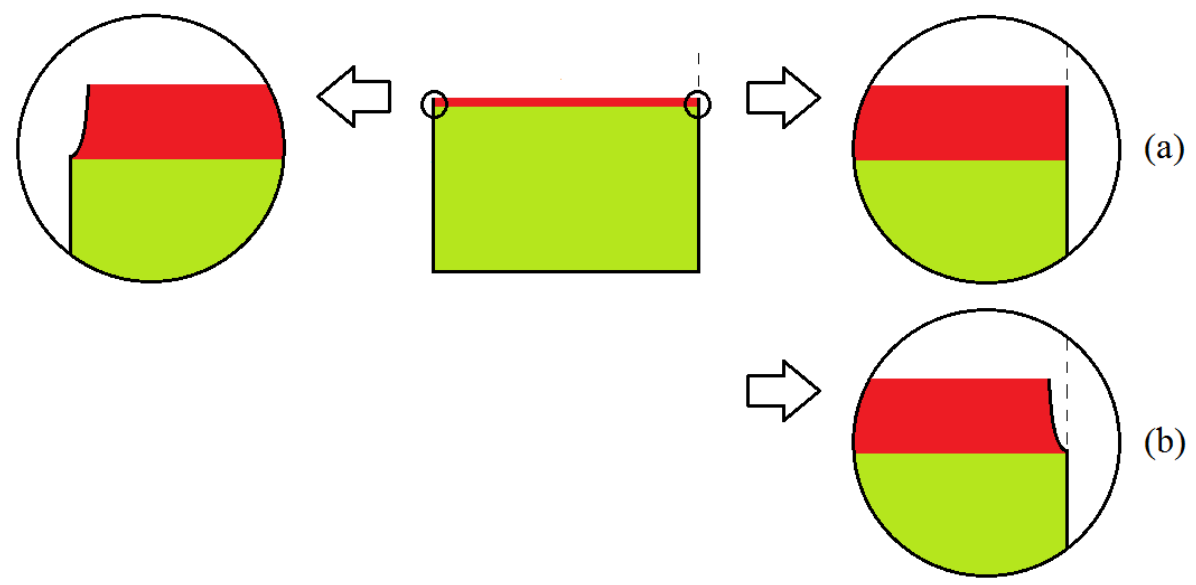

Figure 18. The two states of the new RRVE: (a) prior to the onset of a new family of cracks, (b) following the onset. Again, only one half of the RRVE is represented with a symmetry axis on the right.

Unfortunately, applying this procedure greatly overestimates the density of cracks as a function of the applied load, as observed in Figure 19. Indeed, the order of magnitude of the minimum 
theoretical limit for crack spacing can be as small as $t$ (Thouless, 1990) leading to very high crack densities (from $d \approx 20000 \mathrm{~mm}^{-1}$ for $t=0.043 \mu \mathrm{m}$ to $d \approx 3000 \mathrm{~mm}^{-1}$ for $t=0.32 \mu \mathrm{m}$ ).
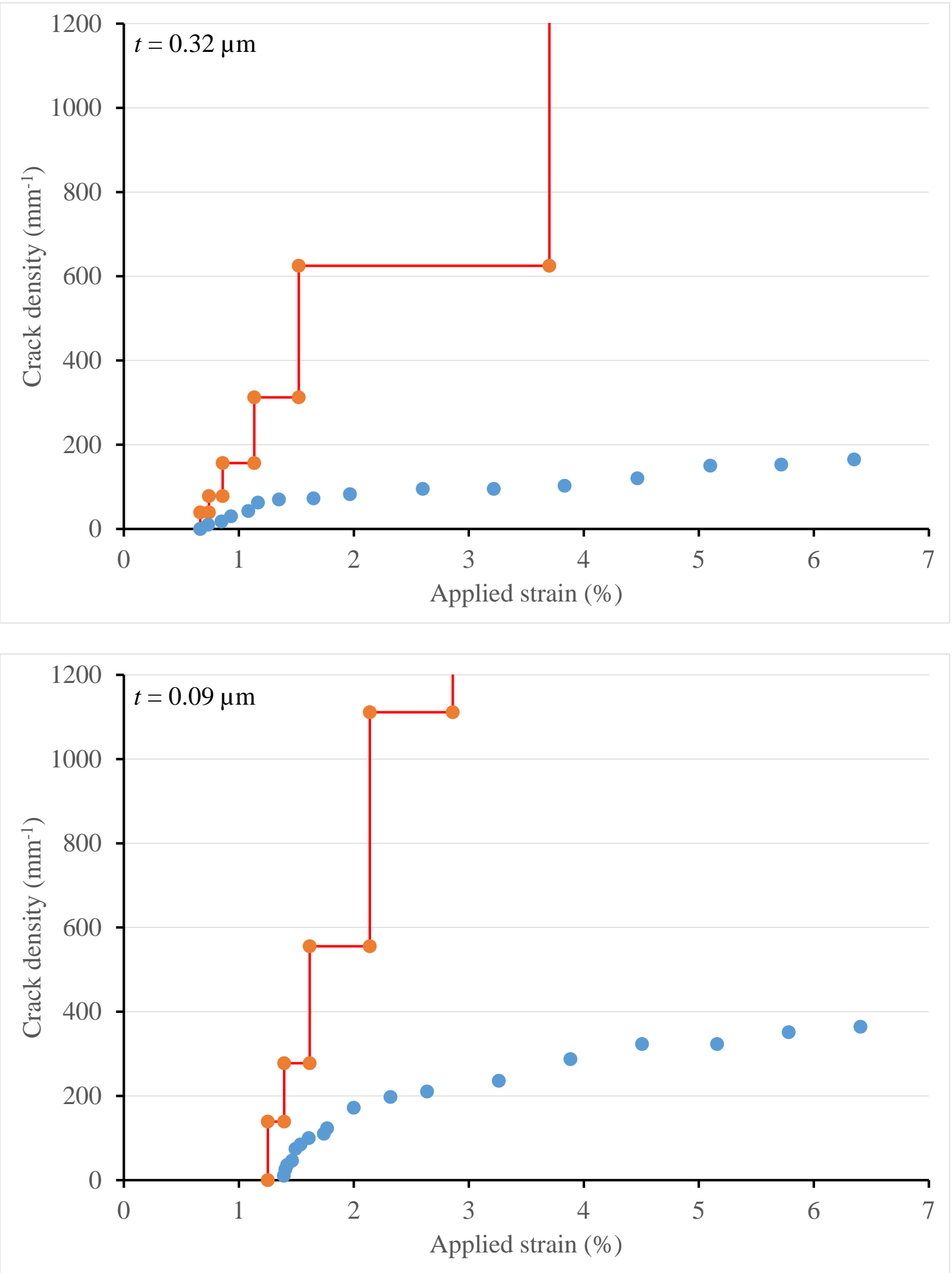


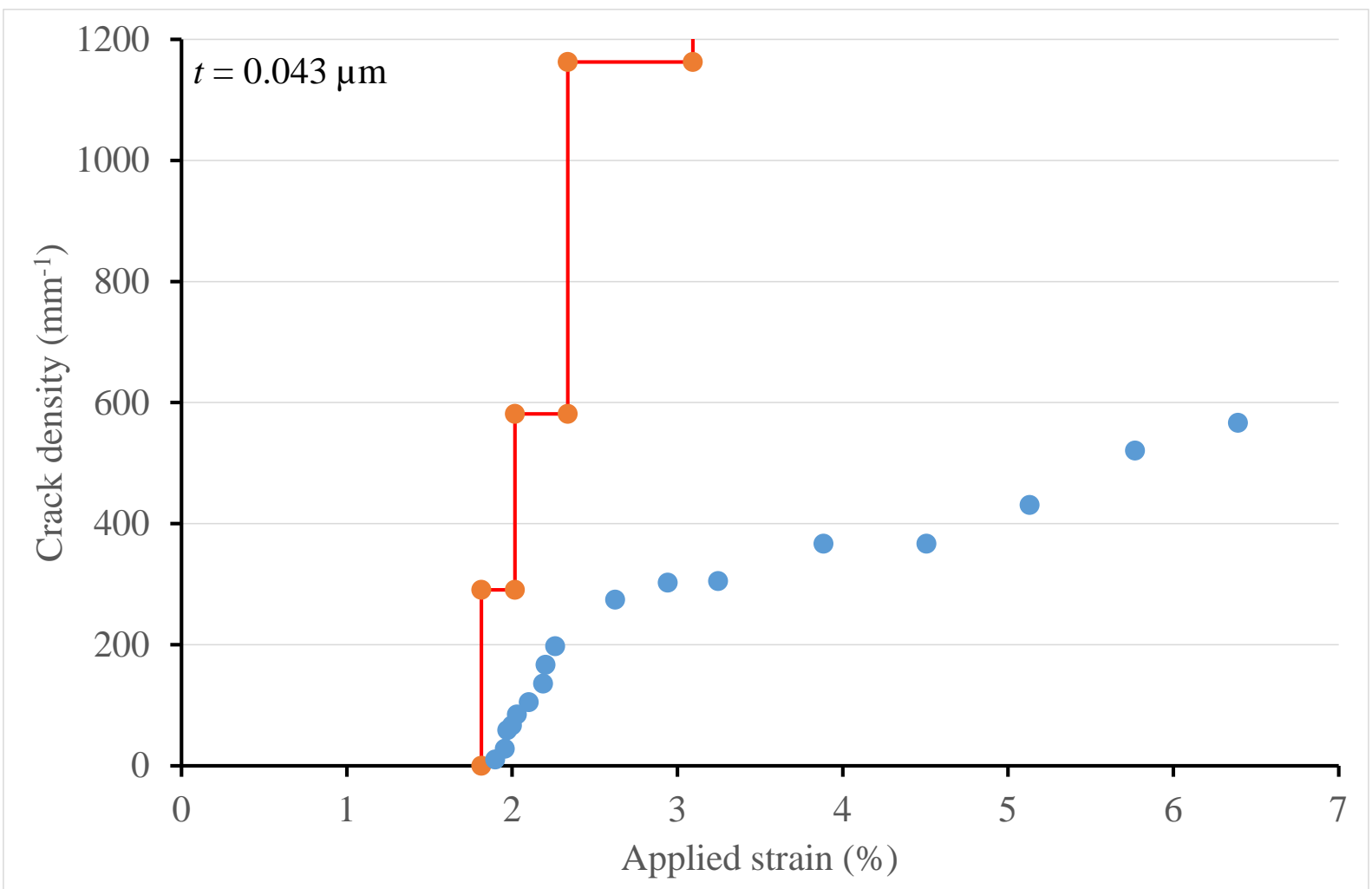

Figure 19. Density of cracks function of the applied strain: comparison between experiments by Hsueh and Yanaka (blue circles) and simulations (red solid line and circles) without delamination, for $t=0.32,0.09,0.043 \mu \mathrm{m}$ (from top to bottom) and $G_{\mathrm{c}}=6 \mathrm{Jm}^{-2}$.

It can be seen in Figure 19 that the only satisfying agreement lies in the critical load at onset of the first family of cracks and the initial density of cracks.

\subsection{Forthcoming crack density function of the applied strain, with delamination}

Actually, the film is stiffer than the substrate and the singularity at the tip of the crack impinging the interface is strong (Leguillon and Sanchez-Palencia, 1992), the singularity exponent is smaller than $1 / 2$, i.e. more harmful than that at the tip of a crack in a homogeneous material. In such a situation, the crack must evolve either penetrating the substrate or growing along the interface (Figure 20) (He and Hutchinson, 1989; Ye et al., 1992; Leguillon and Martin, 2014). The substrate is made of a polymer having in general a high toughness and in any case higher than in the film or along the interface. Thus, based on remarks found in (Jansson et al., 2006a; Jansson et al., 2006b), we make the assumption that the crack is deflected and grows along the interface leading to a film delamination. Such debonding was observed for instance in (Bordet et al., 1998). Note that even if the toughness of the interface is unknown, it cannot be larger than that of the film, otherwise the crack will grow parallel to the interface slightly shifted in the film, leading to a similar film delamination.

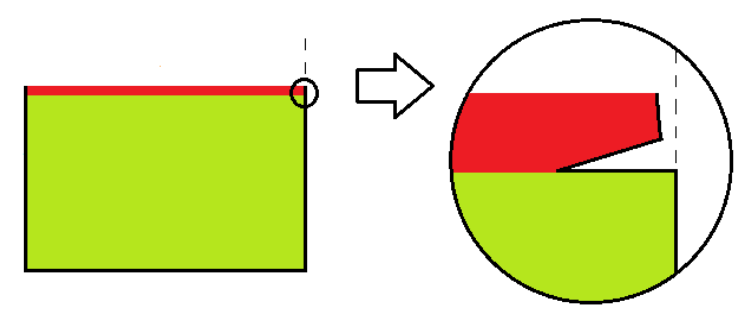

Figure 20. Delamination at the tip of a crack in the RRVE. 
Because of the strong singularity, the growth of the delamination crack is studied using the Griffith criterion based on the energy release rate $G=G_{c}$, note that the interface toughness is taken equal to the film toughness $G_{c}$, according to the above remark. This may lead to underestimating the delamination length if the toughness of the interface is actually lower. Figure 21 shows the delamination length at the tip of the first family of cracks for $t=0.32 \mu \mathrm{m}$, function of the applied load. We recall that it occurs for $\varepsilon^{\mathrm{a}}=0.66 \%$ and the crack half-spacing is $l=12.8 \mu \mathrm{m}(40 \times t)$. According to the strong singularity, the delamination length jumps to 0.1 $\mu \mathrm{m}$ (Figure 21) and then grows in a stable manner.

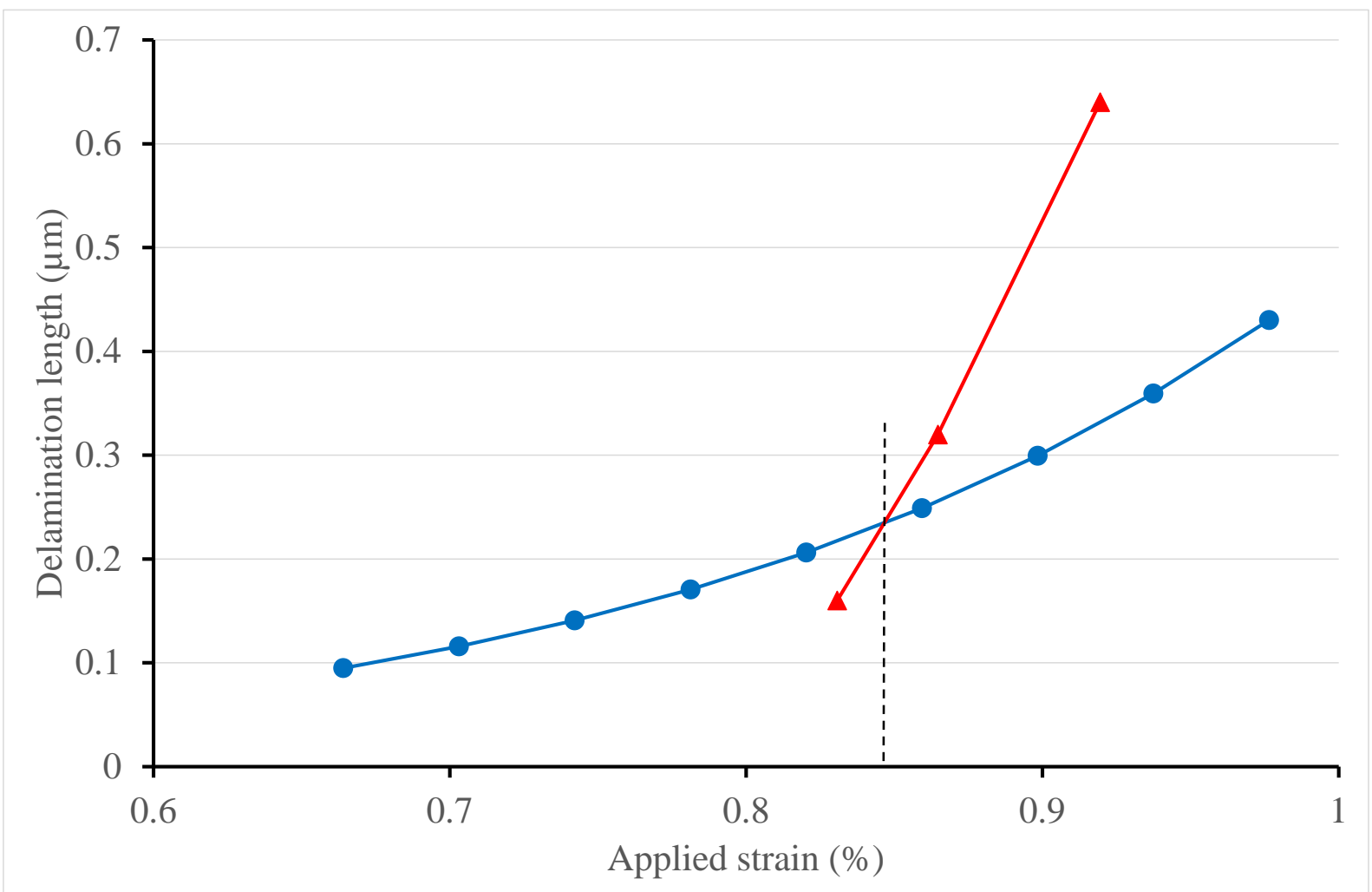

Figure 21. Delamination length at the tip of the first family of cracks function of the applied load (blue solid line and circles), applied strain at the onset of a new family of cracks function of the delamination length (red solid line and triangles), for $t=0.32 \mu \mathrm{m}$. The onset of the first subdivision occurs for an applied strain defined by the intersection of the two curves.

An increasing load causes the growth of the delamination crack but will also lead, at a given value, to the appearance of a new family of cracks. Of course, such value depends strongly on the delamination length, this nonlinear problem is graphically solved. The previous procedure is applied to the RRVE depicted in Figure 22 for different delamination lengths and the critical applied strain triggering a new subdivision is recorded. It is plotted in Figure 21, the solution to the nonlinear problem is at the point where the two curves intersect, i.e. for $\varepsilon^{\mathrm{a}} \approx 0.85 \%$ and a delamination length of $0.24 \mu \mathrm{m}$ (slightly smaller than the film thickness). 


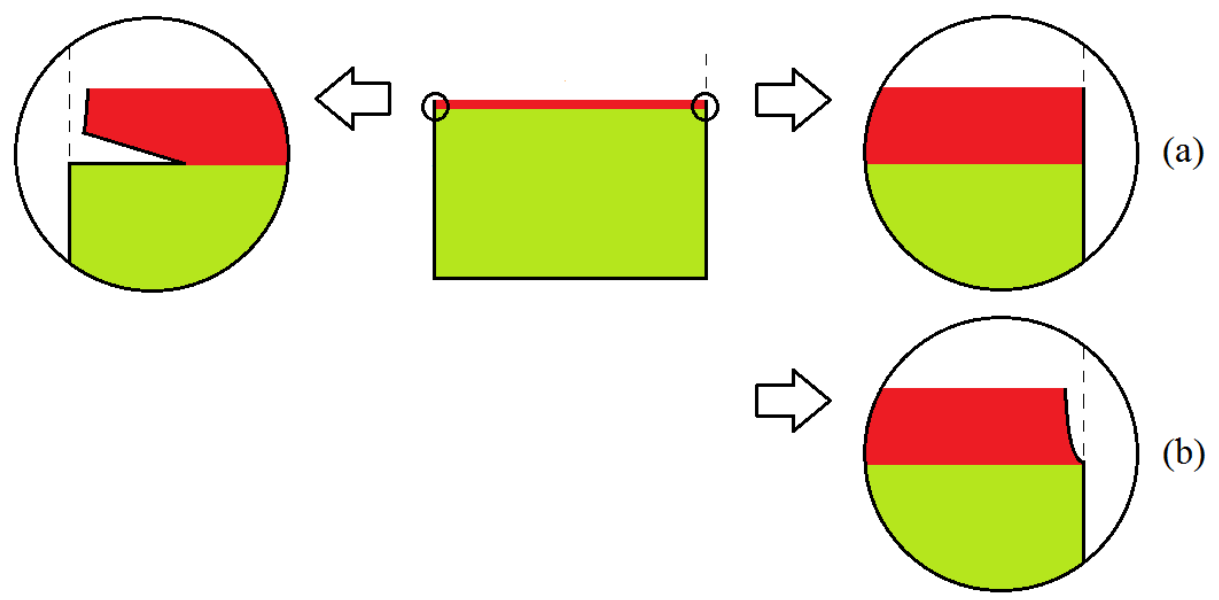

Figure 22. The two states of the RRVE with delamination: (a) prior to the onset of a new family of cracks, (b) following the onset. Only one half of the RRVE is represented with a symmetry axis on the right.

Then, this procedure is repeated for each new subdivision. Nevertheless, it stops after two steps because the nonlinear problem has no longer any solution (Figure 23). Delamination prevails over cracking, its growth is large enough to inhibit the formation of new subdivisions.

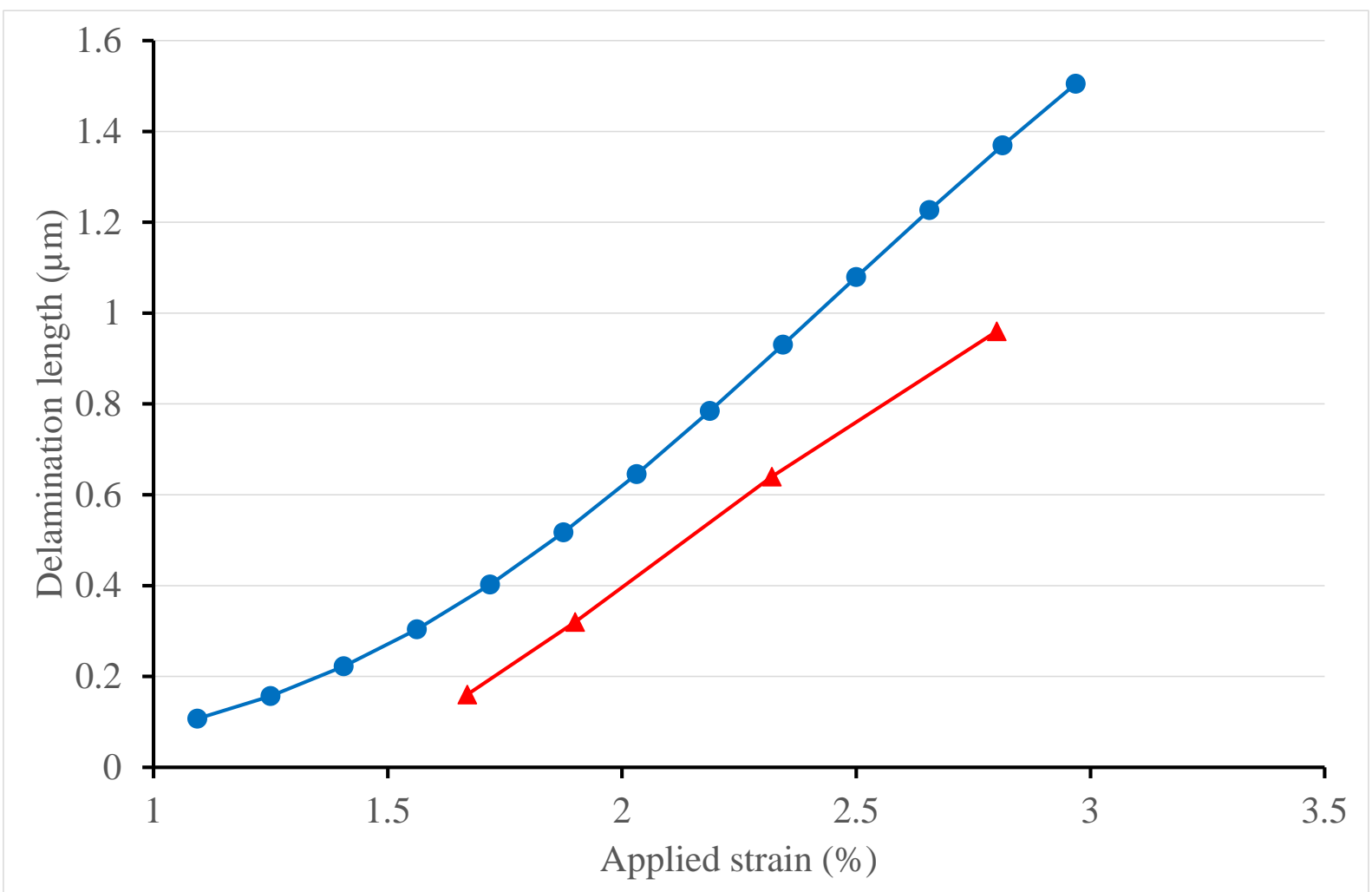

Figure 23. Delamination length at the tip of the third family of cracks function of the applied load (blue solid line and circles), applied strain at the onset of a new family of cracks function of the delamination length (red solid line and triangles), for $t=0.32 \mu \mathrm{m}$. The onset of a new subdivision would occur only if the two curves intersect.

Resulting simulations of the density of cracks function of the applied load are shown in Figure 24 . The agreement is rather good for $t=0.32 \mu \mathrm{m}$, the quality of the prediction is then degraded for decreasing values of film thicknesses, while respecting approximately the orders of 
magnitude. Keep in mind that this is a high estimate because the toughness of the interface was taken equal to that of the film whereas it could be lower (Jansson et al., 2006b). In any case, the gap is not as large as observed in the previous section, and the last horizontal line corresponds to a kind of saturation of the density of cracks, no further subdivision can occur.
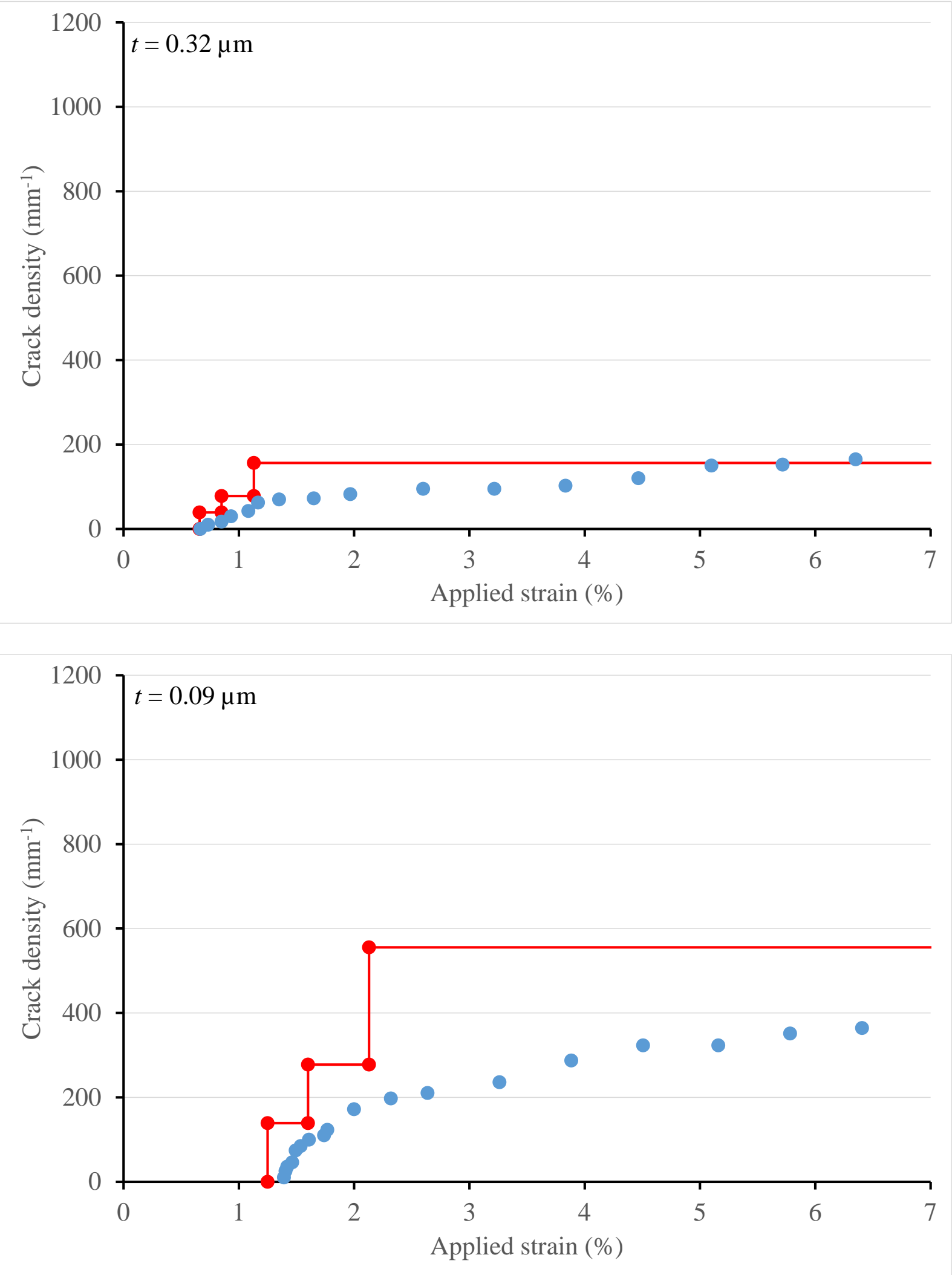


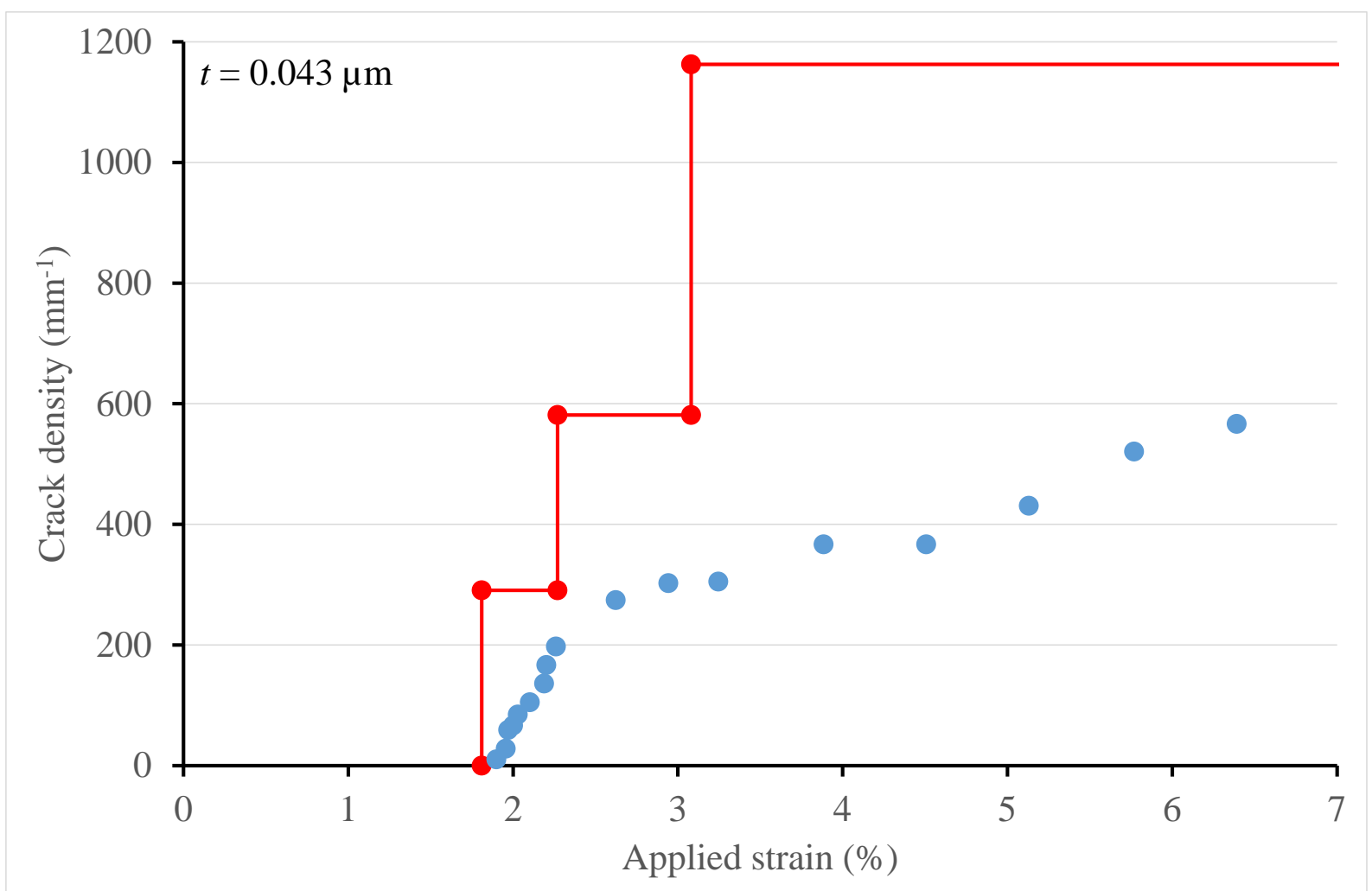

Figure 24. Density of cracks function of the applied strain: comparison between experiments by Hsueh and Yanaka (blue circles) and simulations (red solid line and triangles) with delamination, for $t=0.32,0.09,0.043 \mu \mathrm{m}$ (from top to bottom) and $G_{\mathrm{C}}=6 \mathrm{Jm}^{-2}$.

The use of the RRVE, based on a single geometry (and a single mesh for the FE computations) rescaled for the different film thicknesses, has an important consequence. All the above results can be exhibited on a single master curve by rescaling the actual density of cracks, i.e. the inverse of the cracks spacing, by $t$ and the actual applied strain (varying like $1 / \sqrt{t}$ as already mentioned) by $\sqrt{t}$. This is shown in Figure 25 where the agreement between theory and experiments is much more obvious. 


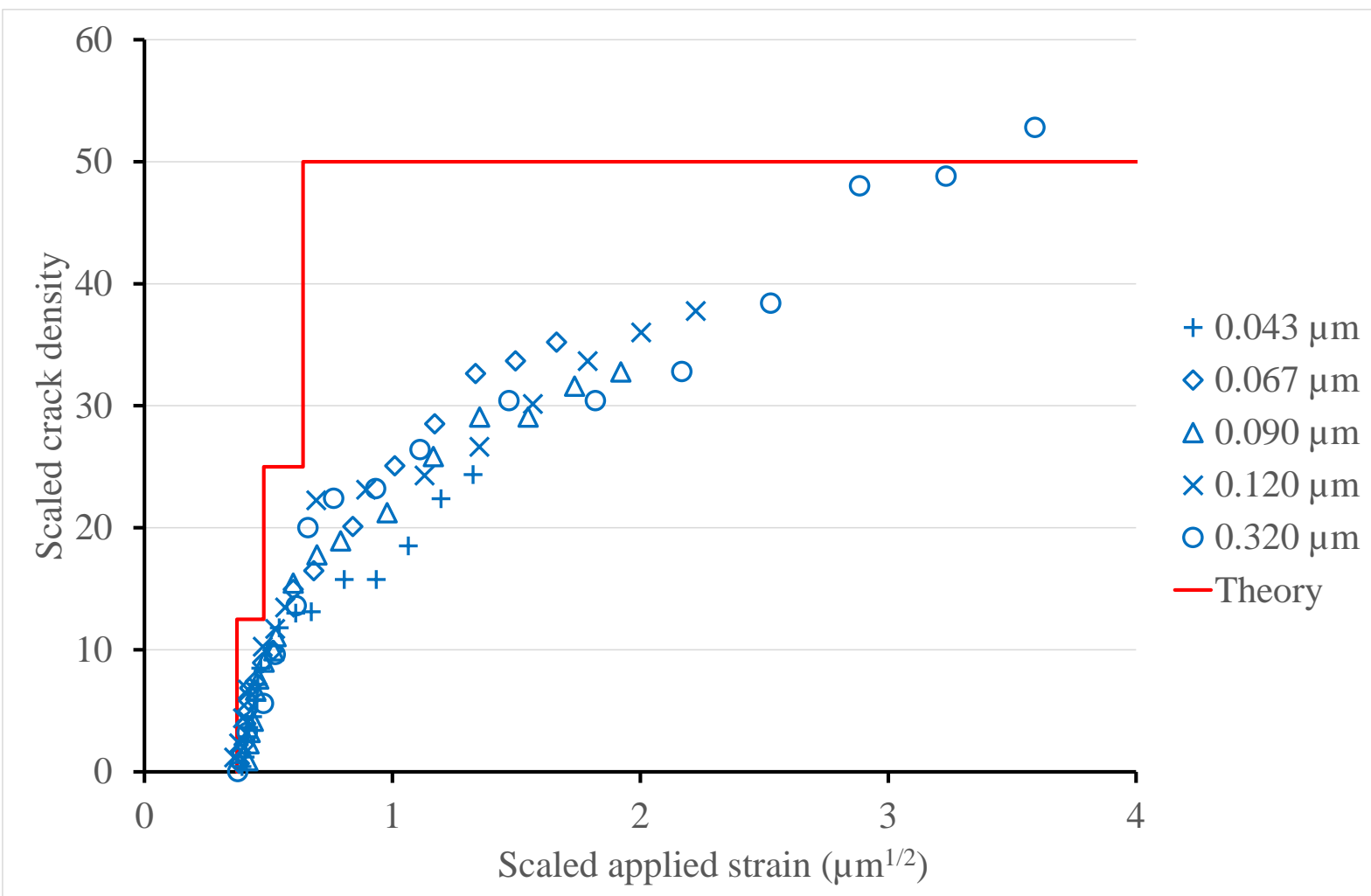

Figure 25. Master curve showing the dimensionless scaled density of cracks vs. the scaled applied strain. The actual density of cracks is multiplied by $t$ (in $\mu \mathrm{m}$ ) and the actual applied strain by $\sqrt{t}$. The solid line is the present theoretical prediction and the symbols correspond to the experiments by Hsueh and Yanaka for different film thicknesses.

Note that, in this subsection, linear elasticity models are used despite the rather high strains involved in the predictions (the last subdivision occurs for strains between 1 and $3.5 \%$ depending on the film thickness). It seems that this is an acceptable approximation which allows the simplified calculations mentioned above.

\section{Conclusion}

The first difficulty linked to the extreme thinness and the brittleness of the films raised a question: does the CC is still working for very thin brittle films? The answer is obviously yes and in addition it allows to decide whether the energy criterion or the stress criterion is governing the multi-cracking phenomenon. For very thin films the energy condition governs the cracking mechanisms while the stress criterion can become predominant for thicker films. The thermal residual stresses play a role in the latter case whereas they can be neglected when the energy condition is governing the cracking process.

The second difficulty to overcome was related to the high density of cracks met in the experiments and a method to address this problem. The use of an RVE has shown to be effective both in the prediction of residual stresses and in the prediction of the critical loading triggering the onset of the first family of cracks. It is even proved that under some conditions a simplified RVE (called RRVE) can be used. It is either when the residual stresses can be neglected, or when the curvature of the specimen is known allowing an accurate determination of the residual stresses. The RRVE approach allows using simplified calculations on a single FE mesh whatever the whole length of the specimen, the height of the substrate and the thickness of the film. New meshes need to be built only when varying the RRVE length, i.e. the crack spacing. 
In addition, this simplified formulation allows showing, when the cracking mechanism is energy driven, that the applied strain at failure varies like the inverse square root of the film thickness. Even better, it allows to evidence the existence of a master curve of the scaled density of cracks function of a scaled applied strain independent of the film thicknesses. Of course, there is no proof to this statement but this could help future research in this topic.

It remains an important issue to discuss, the reliability of the density of cracks prediction and a concomitant question: what is the role of a plastic strain in the substrate? This has been widely discussed in the literature (e.g. Hu and Evans, 1989; Ramsey et al., 1991; Beuth and Klingbeil, 1996; Ambrico and Begley, 2002; Jansson et al., 2006b; Andersons et al., 2008) without a definitive answer. On the one hand, Andersons et al. underline the weak influence of a localized yielding on the critical load triggering the onset of the first family of cracks, when compared to a linear elastic substrate response. On the other hand, Jansson et al. claim that the plastic dissipation within the substrate must be correctly accounted for having realistic interfacial and coating toughness values. Our point of view is that, in agreement with Andersons at al., presumably yielding in the substrate has few influence on the transverse cracking in the film. However, it can play a role in the mechanisms that ensue. Obviously, the interface debonding strongly influences the evolution of crack densities and the plasticity in the substrate will act in the same direction. The influence of substrate plasticity is even more important when the film is thin and stresses that come into play are high. This could further reduce the remaining gap between the measurements and the predictions we have made for the thinner films.

\section{References}

Ambrico J.M., Begley M.R., 2002. The role of initial flaw size, elastic compliance and plasticity in channel cracking of thin films, Thin Solid Films 419, 144-153.

Andersons J., Modniks J., Leterrier Y., Tornare G., Dumont P., Manson J-A.E., 2008. Evaluation of toughness by finite fracture mechanics from crack onset strain of brittle coatings on polymers, Theor. And Appl. Fract. Mech. 49, 151-157.

Beuth J.L., Klingbeil N.W., 1996. Cracking of thin films bonded to elastic-plastic substrates, J. Mech. Phys. of Solids 44, 1411-1428.

Bordet H., Ignat M., Dupeux M., 1998. Analysis of the mechanical response of film on substrate systems presenting rough interfaces, Thin Solid Films 315, 207-213.

Chen Z., Cotterell B., Wang W., 2002. The fracture of brittle thin films on compliant substrates in flexible displays. Engng. Fract. Mech. 69, 597-603.

Crawford G.P., 2005. Flexible flat panel displays, Wiley Series in Display Technology, Crawford Ed., John Wiley \& Sons.

Evans A.G., Hutchinson G.W., 1995. The thermomechanical integrity of thin films and multilayers, Acta metal. Mater. 43, 2507-2530.

Freund L.B., Suresh S., 2004. Thin film materials, Cambridge University Press, Cambridge UK.

Fu Y., Zhang X.C., Xuan F.Z., Tu S.T., Wang Z.D., 2013. Multiple cracking of thin films due to residual stress combined with bending stress. Comp. Mater. Sci. 73, 113-119.

Hashin Z., 1996. Finite thermoelastic fracture criterion with application to laminate cracking analysis, J. Mech. Phys. Solids 44, 1129-1145.

He, M.Y., Hutchinson, J.W., 1989. Crack deflection at an interface between dissimilar elastic materials. Int. J. Solids Structures 25, 1053-1067.

Hsueh C.H., Yanaka M., 2003. Multiple film cracking in film/substrate systems with residual stresses and unidirectional loading, J. Mater. Sci. 38, 1809-1817. 
Hu M.S., Evans A.G., 1989. The cracking and decohesion of thin films on ductile substrates, Acta Metall. 37, 917-925.

Jansson N.E., Leterrier Y., J.-A.E., Manson J.-A.E., 2006a. Modeling of multiple cracking and decohesion of a thin film on a polymer substrate, Engng. Fract. Mech. 73, 2614-2626.

Jansson N.E., Leterrier Y., Medico L., Manson J.A.E., 2006b. Calculation of adhesive and cohesive fracture toughness of a thin brittle coating on a polymer substrate, Thin Solids Films 515, 2097-2105.

Leguillon D., 2002. Strength or toughness? A criterion for crack onset at a notch, Eur. J. Mech. A/Solids 21, 61-72.

Leguillon D., Sanchez-Palencia E., 1992. Fracture in Heterogeneous Materials, Weak and Strong Singularities, in New Advances in Computational Structural Mechanics, P. Ladeveze and O.C. Zienkiewicz eds., Studies in applied mechanics, 32, Elsevier, Amsterdam, 1992, 423-434.

Leguillon D., Martin E., 2014. Crack nucleation at stress concentration points in composite materials - Application to the crack deflection by an interface. In Mathematical Methods and Models in Composites, Computational and Experimental Methods in Structures, V. Mantic Ed., Imperial College Press, London, 401-424.

Leguillon D., Martin E., Sevecek O., Bermejo R., 2015. Application of the coupled stressenergy criterion to predict the fracture behaviour of layered ceramics designed with internal compressive stresses, Eur. J. Mech. A/Solids 54, 94-104.

Leguillon D., Lafarie-Frenot M.C., Pannier Y., Martin E., 2016. Prediction of the surface cracking pattern of an oxidized polymer induced by residual and bending stresses. Int. J. Solids and Structures 91, 89-101.

Leguillon D., Li J., Martin E., 2017. Multi-cracking in brittle thin layers and coatings using a FFM model, Eur. J. Mech. A/Solids 63, 14-21.

Ramsey P.M., Chandler H.W., Page T.F., 1991. Bending test to estimate through-thickness strength and interfacial shear strength in coated systems, Thin Solid Films 201, 81-89.

Thouless M.D., 1990. Crack spacing in brittle films on elastic substrate, J. Am. Ceram. Soc. 73, 2144-2146.

Timm D.H., Guzina B.B., Voller V.R., 2003. Prediction of thermal crack spacing, Int. J. Solids Structures 40, 125-142.

Weissgraeber P., Leguillon D., Becker W., 2016. A review of Finite Fracture Mechanics: Crack initiation at singular and non-singular stress-raisers, Arch. Appl. Mech. 86, 375-401.

Yanaka M., Miyamoto T., Tsukahara Y., Takeda N., 1999. In situ observation and analysis of multiple cracking phenomena in thin glass layers deposited on polymer films, Composite Interfaces 6, 409-424.

Ye T., Suo Z., Evans A.G., 1992. Thin film cracking and the role of substrate and interface, Int. J. Solids Structures 29, 2639-2648.

Zhang X. C., Xuan F. Z., Zhang Y. K. and Tu S. T., 2008. Multiple film cracking in film/substrate systems with mismatch strain and applied strain, J. Appl. Phys. 104, 063520.

\section{Appendix 1}

Special solutions

$E$ is the Young modulus and $v$ the Poisson ratio, the index $\mathrm{f}$ holds for film s for substrate, $l$ is the half length of the RVE.

- Tensile loading 


$$
\left\{\begin{array}{l}
V_{1}^{\mathrm{t}}=\frac{l-x_{1}}{l}(e=1) ; \sigma_{12}^{\mathrm{t}}=\sigma_{22}^{\mathrm{t}}=0 \\
\sigma_{11}^{\mathrm{t}}=\frac{E_{\mathrm{f}}}{1-v_{\mathrm{f}}^{2}} \varepsilon_{11}^{\mathrm{t}}=-\frac{1}{l} \frac{E_{\mathrm{f}}}{1-v_{\mathrm{f}}^{2}} \text { in the film } \\
\sigma_{11}^{\mathrm{t}}=\frac{E_{\mathrm{s}}}{1-v_{\mathrm{s}}^{2}} \varepsilon_{11}^{\mathrm{t}}=-\frac{1}{l} \frac{E_{\mathrm{s}}}{1-v_{\mathrm{s}}^{2}} \text { in the substrate }
\end{array}\right.
$$

- Bending loading

$$
\left\{\begin{array}{l}
V_{1}^{\mathrm{b}}=x_{2} \frac{l-x_{1}}{l}(m=1) ; \sigma_{12}^{\mathrm{b}}=\sigma_{22}^{\mathrm{b}}=0 \\
\sigma_{11}^{\mathrm{b}}=\frac{E_{\mathrm{f}}}{1-v_{\mathrm{f}}^{2}} \varepsilon_{11}^{\mathrm{b}}=-\frac{x_{2}}{l} \frac{E_{\mathrm{f}}}{1-v_{\mathrm{f}}^{2}} \text { in the film } \\
\sigma_{11}^{\mathrm{b}}=\frac{E_{\mathrm{s}}}{1-v_{\mathrm{s}}^{2}} \varepsilon_{11}^{\mathrm{b}}=-\frac{x_{2}}{l} \frac{E_{\mathrm{s}}}{1-v_{\mathrm{s}}^{2}} \text { in the substrate }
\end{array}\right.
$$

- Constrained thermoelastic loading

$$
\left\{\begin{array}{l}
V_{1}^{\mathrm{c}}=0 ; \sigma_{12}^{\mathrm{c}}=\sigma_{22}^{\mathrm{c}}=0 \\
\sigma_{11}^{\mathrm{c}}=0 \text { in the film } \\
\sigma_{11}^{\mathrm{c}}=-\frac{E_{\mathrm{s}}}{1-v_{\mathrm{s}}^{2}} \varepsilon_{11}^{\mathrm{in}}=-\frac{E_{\mathrm{s}}}{1-v_{\mathrm{s}}^{2}} \alpha \Delta \theta \text { in the substrate }
\end{array}\right.
$$

$\Delta \theta$ is the temperature change and $\alpha$ is the coefficient of thermal expansion, it is taken 0 in the film in both examples.

\section{Appendix 2}

The $2 \times 2$ system (16) when there is no crack in the film is 


$$
\left\{\begin{array}{l}
R\left(\underline{V}^{\mathrm{t}}\right)=\frac{1}{l}\left(\frac{E_{\mathrm{s}}}{1-v_{\mathrm{s}}^{2}} h+\frac{E_{\mathrm{f}}}{1-v_{\mathrm{f}}^{2}} t\right) \\
M\left(\underline{V}^{\mathrm{t}}\right)=R\left(\underline{V}^{\mathrm{b}}\right)=\frac{1}{2 l}\left(\frac{E_{\mathrm{f}}}{1-v_{\mathrm{f}}^{2}}-\frac{E_{\mathrm{s}}}{1-v_{\mathrm{s}}^{2}}\right)\left[\left(\frac{h+t}{2}\right)^{2}-\left(\frac{h-t}{2}\right)^{2}\right] \\
M\left(\underline{V}^{\mathrm{b}}\right)=\frac{1}{3 l}\left[\frac{E_{\mathrm{s}}}{1-v_{\mathrm{s}}^{2}}\left(\left(\frac{h+t}{2}\right)^{3}+\left(\frac{h-t}{2}\right)^{3}\right)+\frac{E_{\mathrm{f}}}{1-v_{\mathrm{f}}^{2}}\left(\left(\frac{h+t}{2}\right)^{3}-\left(\frac{h-t}{2}\right)^{3}\right)\right] \\
R\left(\underline{V}^{\mathrm{c}}\right)=h \frac{E_{\mathrm{s}}}{1-v_{\mathrm{s}}^{2}} \alpha \Delta \theta \\
M\left(\underline{V}^{\mathrm{c}}\right)=-\frac{1}{2} t h \frac{E_{\mathrm{s}}}{1-v_{\mathrm{s}}^{2}} \alpha \Delta \theta
\end{array}\right.
$$

$t$ is the film thickness and $h$ the substrate height. 